CU-TP-1076

hep-th/0212328

\title{
ADHMN boundary conditions from removing monopoles
}

\author{
Xingang Chent and Erick J. Weinbergit \\ Physics Department, Columbia University \\ New York, New York 10027
}

\begin{abstract}
Boundary conditions play an important role in the ADHMN construction of BPS monopole solutions. In this paper we show how different types of boundary conditions can be related to each other by removing monopoles to spatial infinity. In particular, we use this method to show how the jumping data naturally emerge. The results can be interpreted in the D-brane picture and provide a better understanding of the derivation of the ADHMN construction from D-branes. We comment briefly on the cases with non-Abelian unbroken symmetry and massless monopoles.
\end{abstract}

*email address: xgchen@phys.columbia.edu

†email address: ejw@phys.columbia.edu 


\section{INTRODUCTION}

The Atiyah-Drinfeld-Hitchin-Manin-Nahm (ADHMN) construction [1, 2] is a powerful method for constructing the Bogomol'nyi-Prasad-Sommerfield (BPS) [3] magnetic monopole solutions in Yang-Mills-Higgs theory. In this method, the problem of solving the BPS equations for gauge and Higgs fields in three-dimensional space is reduced to that of solving ordinary differential equations for a triplet of Hermitian matrices $T_{a}$, called the Nahm data, that are functions of a single variable $t$. We will consider the case of an $\mathrm{SU}(N)$ theory maximally broken to $[\mathrm{U}(1)]^{N-1}$ by an adjoint Higgs field with asymptotic form diag $\left(t_{1}, t_{2}, \ldots, t_{N}\right)$, where $t_{1}<t_{2}<\cdots<t_{N}$ and $\sum t_{i}=0$. There are $N-1$ species of "fundamental monopoles" [4], each carrying a single unit of one of the topological charges. The $T_{a}$ are defined on $t_{1} \leq t \leq t_{N}$. On the interval $t_{i}<t<t_{i+1}$, they are $\left(m_{i} \times m_{i}\right)$-dimensional, where the $m_{i}$ give the numbers of the various fundamental monopoles. We will denote this system as $\left(m_{1}, m_{2}, \ldots, m_{N-1}\right)$.

The boundary conditions are important in this construction. For example, in $\mathrm{SU}(2)$ where we have only one interval $\left(t_{1}, t_{2}\right)$, the Nahm data must have poles at the boundaries $t=t_{1}$ and $t=t_{2}$. For $\mathrm{SU}(N)$, where there are $N-1$ intervals, the boundary conditions at $t_{i}$ depend on the numbers of fundamental monopoles on either side of this boundary. When the numbers are unequal, the boundary conditions are a generalization of those in $\mathrm{SU}(2)$ case. There is an additional element if $m_{i}=m_{i+1}$ for some $i$. In this case we also have "jumping data", consisting of a $2 m_{i}$-component vector $a_{i}$ located at the boundary $t=t_{i}$.

While the constructions for the cases with or without jumping data are different, the following intuitive physical picture suggests that there must be a connection between them. Suppose we consider a solution with $m_{i}>m_{i+1}$. We can gradually deform the solution by removing the extra fundamental monopoles to spatial infinity, so that the number of fundamental monopoles in $\left(t_{i}, t_{i+1}\right)$ becomes equal to $m_{i+1}$. We will study in this paper how the jumping data appear in this process.

More generally, we will show how the ADHMN construction for magnetic charge $\left(m_{1}, \ldots, m_{i}, \ldots, m_{N+1}\right)$ goes over to a "reduced" ADHMN construction for charge $\left(m_{1}, \ldots, m_{i}-1, \ldots, m_{N+1}\right)$ when one of the fundamental monopoles is taken to spatial infinity.

There is a D-brane interpretation of this construction [5, 6, 7,8,9]. In this picture the monopoles are D-strings ending on D3 branes [10,11], $t$ parameterizes the direction parallel to the D-strings, the $t_{i}$ are the places where the D3 branes are located and the Nahm data $T_{a}(t)$ describe the positions and interactions of the various D-string segments. When the gauge symmetry is maximally broken, this configuration can be thought of as a superposition 
of different numbers of fundamental monopoles, with each D-string joining adjacent D3 branes representing a fundamental monopole.

The easiest way to get the jumping data is to start with a D0-D4 system and use Tduality to get the D1-D3 configuration [8]. This requires that the numbers of D-string segments (fundamental monopoles) on both sides of the D3-brane (boundary) are the same. One finds that the jumping data originate from the hypermultiplets that describe the D0-D4 strings. After the T-duality, the hypermultiplets are localized at the positions of the D3branes (boundaries). Therefore, it is interesting to see how these hypermultiplets (jumping data) appear or disappear when we remove D-string segments (fundamental monopoles). This will help us to understand how to consistently derive the other boundary conditions.

This paper is organized as follows: In Sec. II, we briefly review the ADHMN construction for $\mathrm{SU}(N)$. In Sec. III, we study the case of $\mathrm{SU}(2)$. We show how the $k$-monopole ADHMN construction becomes equivalent to the reduced $(k-1)$-monopole construction when one monopole is removed to spatial infinity. In Sec. IV], we study cases in larger groups where no jumping data is involved. In Sec. $\square$, we consider the cases $m_{i}=m_{i+1}+1$ and $m_{i}=m_{i+1}$, where jumping data appears or disappears in the reduced problem. An explicit SU(3) example is given in Sec. $\nabla 1$. The results are interpreted in terms of the D-brane picture in Sec. VII. Section VIII contains the conclusions and some discussion. Some details omitted from Sec. $\mathbb{E A}$ are included in Appendix A.

\section{THE ADHMN CONSTRUCTION}

In this section, we briefly outline the ADHMN construction. We restrict our discussion to the $\mathrm{SU}(N)$ case [12]. The generalization to $\mathrm{SO}(N)$ and $\operatorname{Sp}(N)$ is discussed in 12. There are basically three steps in the ADHMN construction. We first illustrate this in the SU(2) case with $m$ fundamental monopoles and asymptotic Higgs field $\operatorname{diag}\left(t_{1}, t_{2}\right)$.

The first step is to find the Nahm data, which are $m \times m$ Hermitian matrices $T_{a}(t)$ $(a=1,2,3)$ defined on the interval $t_{1} \leq t \leq t_{2}$. They satisfy the Nahm equations 1

$$
\frac{d T_{a}}{d t}=-i \epsilon_{a b c} T_{b} T_{c}
$$

It is useful to note that the Nahm equation is preserved under a unitary transformation $T_{a} \rightarrow U T_{a} U^{\dagger}$, where $U$ is $t$ independent. The boundary conditions are that the $T_{a}$ have simple poles at $t_{1}$ and $t_{2}$, with the residues being $m$-dimensional irreducible $\mathrm{SU}(2)$ representations.

\footnotetext{
${ }^{1}$ Repeated indices $a, b, c$ are summed over unless otherwise indicated. Here, and for most of the paper, we set the gauge group coupling $e=1$.
} 
The second step is to solve the construction equation

$$
0=\left[-\frac{d}{d t}+\left(-T_{a}+r_{a}\right) \otimes \sigma_{a}\right] v_{p}\left(t, r_{a}\right)
$$

where the $\sigma_{a}$ are the Pauli matrices, the $v_{p}$ are $2 m$-component vectors and $p$ labels the linearly independent solutions. We note that the $v_{p}$ depend on the spatial position $\boldsymbol{r}$, while the Nahm data $T_{a}$ do not; this $\boldsymbol{r}$ dependence will often not be explicitly indicated. As we will see shortly, in the $\mathrm{SU}(2)$ case there are two linearly independent $v_{p}$. We normalize these so that

$$
\int d t v_{p}^{\dagger} v_{q}=\delta_{p q}
$$

In the third step we obtain the monopole gauge and Higgs fields satisfying the self-dual BPS equations. If we assemble all the independent normalizable solutions into a $2 m_{i} \times 2$ matrix $\boldsymbol{v}$, then

$$
\begin{aligned}
& \Phi=\int d t t \mathbf{v}^{\dagger} \mathbf{v} \\
& \mathbf{A}=-i \int d t \mathbf{v}^{\dagger} \nabla \mathbf{v} .
\end{aligned}
$$

The linearly independent solutions of the construction equation, Eq. (2.2), can be counted as follows: At each boundary, due to the irreducible SU(2)-valued residue of the Nahm data, one can show that of the $2 m$ solutions near each boundary, $m+1$ behave as $\mid t-$ $\left.t_{\text {end }}\right|^{(m-1) / 2}$, while the other $m-1$ behave as $\left|t-t_{\text {end }}\right|^{-(m+1) / 2}$ and are thus non-normalizable. Matching the $m+1$ normalizable solutions from the left boundary and the $m+1$ normalizable solutions from the right in the middle of the interval imposes $2 m$ constraints, because these vectors are $2 m$-dimensional. This leaves two independent normalizable solutions and thus give the $\mathrm{SU}(2)$ fields in Eq. (2.4). (Although the case $m=1$ has no poles, the counting is the same.)

For the general $\mathrm{SU}(N)$ case with asymptotic Higgs field $\operatorname{diag}\left(t_{1}, t_{2}, \ldots, t_{N}\right)$, the gauge symmetry is broken to $[\mathrm{U}(1)]^{N-1}$. Each $\mathrm{U}(1)$ factor is associated with a fundamental monopole that can be obtained by embedding the unit $\mathrm{SU}(2)$ monopole.

On the interval $\left(t_{i}, t_{i+1}\right)$ the Nahm data are $m_{i} \times m_{i}$ matrices $T_{a}^{i}$ obeying Eq. (2.1). These define a construction equation for $2 m_{i}$-component vectors $v_{p}^{i}$. The boundary conditions at $t_{1}$ and $t_{N}$ are the same as for $\mathrm{SU}(2)$. At the other boundaries, the boundary conditions depend on the number of fundamental monopoles on either side of the boundary.

The $m_{i} \neq m_{i+1}$ case is a generalization of the $\mathrm{SU}(2)$ case. We first assume $m_{i} \equiv$ $m_{i+1}+k>m_{i+1}$. Near the boundary $t_{i+1}^{-}$, the $T_{a}^{i}$ take the form 


$$
\left(\begin{array}{cc}
S_{a} & \mathcal{O}\left(\left(t-t_{i+1}\right)^{(k-1) / 2}\right) \\
\mathcal{O}\left(\left(t-t_{i+1}\right)^{(k-1) / 2}\right) & -\frac{J_{a}^{(k)}}{t-t_{i+1}}
\end{array}\right),
$$

where $S_{a}\left(t_{i+1}\right)=T_{a}^{i+1}\left(t_{i+1}\right)$, and the $J_{a}^{(k)}$ are $k$-dimensional irreducible representations of $\mathrm{SU}(2)$. For the solutions of Eq. (2.2), the upper $2 m_{i+1}$ components of $v_{p}^{i}$ from $\left(t_{i}, t_{i+1}\right)$ are continuous across the boundary, connecting with the $2 m_{i+1}$-component solutions from $\left(t_{i+1}, t_{i+2}\right)$. The other $2 k$ components of $v_{p}^{i}$ from $\left(t_{i}, t_{i+1}\right)$ are finite and terminate at the boundary. The case $m_{i}<m_{i+1}$ is completely analogous.

In the case of $m_{i}=m_{i+1}$, the $T_{a}^{i}$ are discontinuous at the boundary $t_{i+1}$. These discontinuities are described by an extra term involving $2 m_{i+1}$-dimensional row vectors $a_{r \alpha}^{i+1}$, where $\alpha=1,2$ are spinor indices and $r=1, \ldots, m_{i+1}$ :

$$
\begin{aligned}
\left(\Delta T_{a}\right)_{r s} & \equiv T_{a}^{i+1}\left(t_{i+1}\right)_{r s}-T_{a}^{i}\left(t_{i+1}\right)_{r s} \\
& =-\frac{1}{2} a_{s \alpha}\left(\sigma_{a}\right)_{\alpha \beta} a_{r \beta}^{\dagger} \\
& =-\frac{1}{2} \operatorname{tr}_{2}\left(a_{r}^{\dagger} a_{s} \sigma_{a}\right) .
\end{aligned}
$$

[For simplicity we have dropped the superscript $(i+1)$ on $a$.] The trace in the last equality is over the two-dimensional spinor indices $\alpha$ of $a$ and the Pauli matrices $\sigma_{a}$. Correspondingly, the solutions of the construction equations are also discontinuous at the boundary, with

$$
\Delta v=v^{i+1}\left(t_{i+1}\right)-v^{i}\left(t_{i+1}\right)=-a^{(i+1) \dagger} S^{i+1},
$$

where the $S^{i+1}$ are complex numbers.

We can count the number of linearly independent normalizable solutions of the construction equations by a method similar to that for the $\mathrm{SU}(2)$ case. In the $k=0$ case, it is important to note that there is an additional degree of freedom from the vector $a^{i \dagger} S^{i}$ in Eq. (2.7) when we connect the solutions from both side of the boundary. The final result is always $N$.

If we assemble the $S_{p}^{i}$ into an $N$-component row vector $\mathbf{S}_{i}$, the normalization condition Eq. (2.3) becomes

$$
I=\int d t \mathbf{v}^{\dagger} \mathbf{v}+\sum_{i} \mathbf{S}_{i}^{\dagger} \mathbf{S}_{i}
$$

Equation (2.4) becomes

$$
\begin{aligned}
& \Phi=\int d t \mathbf{v}^{\dagger} \mathbf{v}+\sum_{i} t_{i} \mathbf{S}_{i}^{\dagger} \mathbf{S}_{i} \\
& \mathbf{A}=-i \int d t \mathbf{v}^{\dagger} \nabla \mathbf{v}-i \sum_{i} \mathbf{S}_{i}^{\dagger} \nabla \mathbf{S}_{i} .
\end{aligned}
$$


In these equations the sum over $i$ is restricted to the boundaries with $m_{i-1}=m_{i}$.

\section{THE SU(2) EXAMPLE}

We first study the simplest example, that of $\mathrm{SU}(2)$ broken to $\mathrm{U}(1)$, which does not involve the appearance or disappearance of the jumping data. Parameter counting and other analyses [14] suggest that in this case if all the monopoles are separated much further than their core sizes, the solution can be approximated as a superposition of many unit monopoles.

Let us assume that we have $k$ unit monopoles. The Nahm data for this system are $k \times k$ Hermitian matrices $T_{a}$ on the interval $\left(t_{1}, t_{2}\right)$. We want to show that by removing one unit monopole, the $k$ dimensional ADHMN construction effectively becomes that for $k-1$ monopoles. Doing this is also an explicit demonstration of the above mentioned superposition picture. We assume that $k-1$ of the monopoles, as well as the position $\boldsymbol{r}$ where we probe the fields, are located within a region of size $l$, and that the $k$ th monopole is removed by a distance $D \gg l$, which without loss of generality we can take to be along the $z$-axis.

The Nahm data have poles near the boundaries. This requires

$$
T_{a} \approx-\frac{J_{a}^{(k)}}{t-t_{2}}
$$

in the region $\left(t_{2}-1 / D, t_{2}\right)$, and

$$
T_{a} \approx-\frac{\tilde{J}_{a}^{(k)}}{t-t_{1}}
$$

in the region $\left(t_{1}, t_{1}+1 / D\right)$, where $J_{a}^{(k)}$ and $\tilde{J}_{a}^{(k)}$ are $k$-dimensional irreducible representation of $\mathrm{SU}(2)$.

Away from the boundaries, moving one fundamental monopole faraway makes one of the eigenvalues in the Nahm data $T_{a}$ much larger than the others. Therefore the Nahm data in the middle of the interval can be put into the form

$$
T_{a}=\left(\begin{array}{cc}
M_{a} & A_{a}^{\dagger} \\
A_{a} & b_{a}
\end{array}\right),
$$

\footnotetext{
${ }^{2}$ The $J_{a}^{(k)}$ and $\tilde{J}_{a}^{(k)}$ do not have to be the same representation, although they will of course be unitarily equivalent.
} 
where the $M_{a}$ are $(k-1) \times(k-1)$ dimensional Hermitian matrices with entries that are $\mathcal{O}(l)$, the $A_{a}^{\dagger}$ are $k-1$ dimensional vectors that are at most $\mathcal{O}(\sqrt{l D})$, and $b_{a}=\delta_{a 3} D+\mathcal{O}(l)$.

It is useful to note that a unitary transformation $T_{a} \rightarrow U T_{a} U^{\dagger}$ with

$$
U=\left(\begin{array}{cc}
I_{(k-1) \times(k-1)}+\mathcal{O}(l / D) & -\mathcal{K}^{\dagger} / D \\
\mathcal{K} / D & 1+\mathcal{O}(l / D)
\end{array}\right)
$$

and $\mathcal{K} \leq \mathcal{O}(\sqrt{l D})$ shifts

$$
A_{a} \rightarrow A_{a}-\mathcal{K} \delta_{a 3}, \quad M_{a} \rightarrow M_{a}-\frac{\mathcal{K}^{\dagger} \mathcal{K}}{D} .
$$

[In Eq. (3.5) we have omitted terms that vanish when $D \rightarrow \infty$.] By making use of such a transformation, we can always subtract a $t$-independent constant from $A_{3}$. We will use this freedom to make $A\left[\left(t_{1}+t_{2}\right) / 2\right]$ vanish, up to exponentially small terms.

In the following we will show that, in this case, the $A_{a}$ terms are effectively negligible and that the $M_{a}$ obey the Nahm equations for the $k-1$ monopole problem. For $\boldsymbol{r} \sim \mathcal{O}(l)$, the fields derived from $M_{a}$ using Eq. (2.4) approximate those derived from $T_{a}$.

We define $A=A_{1}+i A_{2}, \hat{A}=A_{1}-i A_{2}, M=M_{1}+i M_{2}, \hat{M}=M_{1}-i M_{2}, b=b_{1}+i b_{2}$ and $\hat{b}=b_{1}-i b_{2}$. The Nahm equations separate into the following equations:

$$
\begin{aligned}
\frac{d M_{a}}{d t} & =-i \epsilon_{a b c}\left(M_{b} M_{c}+A_{b}^{\dagger} A_{c}\right), \\
\frac{d A}{d t} & =-A M_{3}+A_{3} M-b A_{3}+b_{3} A, \\
\frac{d \hat{A}}{d t} & =\hat{A} M_{3}-A_{3} \hat{M}+\hat{b} A_{3}-b_{3} \hat{A}, \\
\frac{d A_{3}}{d t} & =\frac{1}{2}(A \hat{M}-\hat{A} M+b \hat{A}-\hat{b} A), \\
\frac{d b}{d t} & =-A A_{3}^{\dagger}+A_{3} \hat{A}^{\dagger}, \\
\frac{d \hat{b}}{d t} & =\hat{A} A_{3}^{\dagger}-A_{3} A^{\dagger}, \\
\frac{d b_{3}}{d t} & =\frac{1}{2}\left(A A^{\dagger}-\hat{A} \hat{A}^{\dagger}\right) .
\end{aligned}
$$

We first consider the middle of the interval, away from the boundaries, where the $M_{a}$ are $\mathcal{O}(l)$. Equation (3.9) gives

$$
A_{3}=\mathcal{O}(l)\left|t_{2}-t_{1}\right| \operatorname{Max}(A, \hat{A}) .
$$

(Recall that we have used a unitary transformation to subtract a constant from $A_{3}$.) 
If $A \gtrsim \hat{A}$, and $D \gg\left(t_{2}-t_{1}\right) \mathcal{O}\left(l^{2}\right)$, the fourth term dominates the right-hand side of Eq. (3.7). This gives

$$
A=C e^{-D\left|t-t_{2}\right|}
$$

where the coefficient $C$ is $t$-independent. Matching this behavior to the pole region at $t \approx t_{2}-1 / D$ requires that $C=\mathcal{O}(D)$. Consequently, Eq. (3.9) gives

$$
A_{3}=C_{3} e^{-D\left|t-t_{2}\right|}
$$

where the coefficient $C_{3}$ is $\mathcal{O}(l)$. Equation (3.8) then implies

$$
\hat{A}=\mathcal{O}\left(l^{2} / D\right) e^{-D\left|t-t_{2}\right|} .
$$

If instead $\hat{A} \gtrsim A$,

$$
\begin{aligned}
\hat{A} & =\hat{C} e^{-D\left|t-t_{1}\right|}, \\
A_{3} & =C_{3} e^{-D\left|t-t_{1}\right|}, \\
A & =\mathcal{O}\left(l^{2} / D\right) e^{-D\left|t-t_{1}\right|},
\end{aligned}
$$

where $\hat{C}$ is $\mathcal{O}(D)$ and $C_{3}$ is $\mathcal{O}(l)$. Equations (3.14 3.16) apply for $t$ closer to $t_{2}$, while Eqs. (3.173.19) apply for $t$ closer to $t_{1}$.

From Eqs. (3.14 3.19), we see that $A_{a} \ll \mathcal{O}(l)$ except within narrow regions of width $t_{D} \equiv \frac{1}{D} \ln \frac{D}{l}$ near the boundaries. Hence, outside these boundary regions, Eq. (3.6) can be approximated by the $(k-1)$-monopole Nahm equations with Nahm data $M_{a}$. In order to match the pole behavior of the $T_{a}$ in $(-1 / D, 0)$ in the original problem, the $(k-1)$-monopole Nahm data $M_{a}$ must have pole behavior in $\left(-1 / l,-t_{D}\right)$. The residues of the poles will be irreducible $\mathrm{SU}(2)$ representations.

To see what happens to the construction equation, we decompose

$$
v=\left(\begin{array}{l}
w \\
z
\end{array}\right),
$$

where $w$ is a $2 m$-dimensional vector and $z$ is 2 -dimensional. The construction equation then becomes

\footnotetext{
${ }^{3}$ Since later we will know that $A_{3} \sim e^{-D\left|t-t_{2}\right|}$ or $e^{-D\left|t-t_{1}\right|}$, this is an over-estimate. In fact, we can replace $\left(t_{2}-t_{1}\right)$ by $1 / D$; i.e., it is enough that $D \gg l$.
} 


$$
\begin{aligned}
& 0=-\frac{d}{d t} w+\left[\left(-M_{a}+r_{a}\right) \otimes \sigma_{a}\right] w-\left(A_{a}^{\dagger} \otimes \sigma_{a}\right) z, \\
& 0=-\frac{d}{d t} z-\left(A_{a} \otimes \sigma_{a}\right) w+\left[\left(-b_{a}+r_{a}\right) \otimes \sigma_{a}\right] z .
\end{aligned}
$$

In the interval $\left(t_{1}+t_{D}, t_{2}-t_{D}\right)$ the $w$ and $z$ components are decoupled, since the contributions to them from the cross terms in Eqs. (3.21) and (3.22) are negligible due to the exponential smallness of the $A_{a}$. There are three types of solutions. The first two types are associated with the reduced Nahm data $M_{a}$. One is of the form

$$
v=\left(\begin{array}{c}
v_{i} \\
0
\end{array}\right)+\cdots, \quad i=1,2
$$

where $v_{i}$ is a normalizable solution of the $(k-1)$-monopole construction equation formed from the $M_{a}$, and the dots represent exponentially small terms. Next are solutions of the form

$$
v=\left(\begin{array}{c}
u_{j} \\
0
\end{array}\right)+\cdots, \quad j=1, \ldots, 2 k-4
$$

where the $u_{j}$ are non-normalizable solutions of the construction equation formed from the $M_{a}$. These behave as $\left|t-t_{\text {end }}\right|^{-k / 2}$ at least near one boundary. Finally, there are two solutions of the form

$$
v=\left(\begin{array}{c}
0 \\
z_{ \pm}
\end{array}\right)
$$

where $z_{ \pm}=e^{ \pm D t} \eta_{ \pm}$and $\sigma_{3} \eta_{ \pm}=\mp \eta_{ \pm}$, and higher order exponential terms are ignored. As we can see, these are concentrated near the boundaries.

We normalize the $v_{i}$ so that

$$
\int v_{i}^{\dagger} v_{j}=\delta_{i j}
$$

and fix the scale in Eqs. (3.24) and (3.25) by requiring

$$
\begin{aligned}
\operatorname{Max}\left[u_{j}\left(t_{1}+1 / D\right), u_{j}\left(t_{2}-1 / D\right)\right] & \sim \sqrt{D}, \\
\operatorname{Max}\left[z_{ \pm}\left(t_{1}+1 / D\right), z_{ \pm}\left(t_{2}-1 / D\right)\right] & \sim \sqrt{D} .
\end{aligned}
$$

From the discussion below Eq. (2.4), we see that $v_{i}\left(t_{1}+1 / D\right)$ and $v_{i}\left(t_{2}-1 / D\right)$ are both of order $D^{-k / 2+1}$.

Now we match the linear combination 


$$
v=\sum_{i=1}^{2} c_{i} v_{i}+\sum_{i=1}^{2 k-4} d_{i} u_{i}+\sum_{i= \pm} e_{i} z_{i}
$$

to the normalizable solutions in the boundary regions $\left(t_{1}, t_{1}+1 / D\right)$ and $\left(t_{2}-1 / D, t_{2}\right)$. As we have seen, within each of these two boundary regions there are $k+1$ normalizable solutions behaving as $\left|t-t_{\text {end }}\right|^{(k-1) / 2}$. The matching of the solutions of Eq. (3.29) on to the boundary region solutions requires that all three terms in Eq. (3.29) be of the same order of magnitude at $\left|t-t_{\text {end }}\right|=1 / D$. This implies that the $d_{i}$ and $e_{i}$ are order of $D^{-(k-1) / 2}$ smaller than the $c_{i}$. Therefore, the last two terms of Eq. (3.29) are negligible 1 in the integrals in the normalization condition, Eq. (2.3), and the fields, Eq. (2.4), since they only give corrections of order $D^{-k+1}$. The changes of the solutions of Eq. (3.23) within $\left(t_{1}, t_{1}+t_{D}\right)$ and $\left(t_{2}-t_{D}, t_{2}\right)$ also only have negligible effect on the fields and normalization condition, because the integration region is too small. So we effectively recover the reduced $(k-1)$ monopole construction.

\section{THE POLE BEHAVIOR}

In this and the next section, we describe how the boundary conditions in the ADHMN construction change when a monopole is removed to spatial infinity. We will concentrate on the case of $\mathrm{SU}(3)$ with magnetic charge $(m+k, m)$, focusing on the boundary between the intervals $\left(t_{1}, t_{2}\right)$ and $\left(t_{2}, t_{3}\right)$; the extension to larger unitary groups is straightforward. We write the $(m+k) \times(m+k)$ Hermitian matrices on the left of $t_{2}$ as $T_{a}^{L}$ and the $m \times m$ Hermitian matrices on the right as $T_{a}^{R}$. Because we will remove one fundamental monopole corresponding to the left interval $\left(t_{1}, t_{2}\right)$, one eigenvalue of $T_{a}^{L}$ should be of order $D$ throughout most of this interval. The other elements of $T_{a}^{L}$ and all the elements of $T_{a}^{R}$ should be $\mathcal{O}(l)$ outside the boundary regions. For simplicity, we take $t_{2}=0$ in this and the next section.

We first study the two simpler cases where no jumping data is involved. These are the case $k>1$ (in Sec. IVA) and the case $k<0$ (in Sec. IVB). In the next section we study how removing a monopole leads to the appearance (for $k=1$ ) and disappearance (for $k=0$ ) of jumping data.

\footnotetext{
${ }^{4}$ Simply stated, the solutions in Eqs. (3.24) and 3.25) share the feature that they decay quickly away from the boundaries, so if their scales are fixed to be finite near the boundary, they do not contribute to the fields. We will see similar situations later.
} 


\section{A. $k>1$ : Reducing the dimension of the pole term on the left}

We first consider the case where we remove one fundamental $(1,0)$ monopole from the $(m+k, m)$ system, with $k>0$. (The discussion of Sec. III can be viewed as the special case where $m=0$.)

We first phrase the problem and our expectation. In the original $(m+k, m)$ problem, we have on the left interval Hermitian matrices $T_{a, \text { orig }}^{L}$ that have one large eigenvalue in the middle of the interval and that develop the poles in the boundary region $(-1 / D, 0)$ of the form

$$
T_{a, \text { orig }}^{L}=\left(\begin{array}{cc}
N_{a} & \mathcal{O}\left(t^{(k-1) / 2}\right) \\
\mathcal{O}\left(t^{(k-1) / 2}\right) & -\frac{J^{(k)}}{t}
\end{array}\right),
$$

where the $N_{a}(0)=T_{a}^{R}(0)$ are $m \times m$ dimensional. We want to show that this is equivalent to the reduced $(m+k-1, m)$ problem whose Nahm data $T_{a \text {,red }}^{L}$ have poles in the boundary region $(-1 / l, 0)$ of the form

$$
T_{a, \text { red }}^{L}=\left(\begin{array}{cc}
N_{a} & \mathcal{O}\left(t^{(k-2) / 2}\right) \\
\mathcal{O}\left(t^{(k-2) / 2}\right) & -\frac{J^{(k-1)}}{t}
\end{array}\right) .
$$

Again, the $N_{a}(0)=T_{a}^{R}(0)$ are $m \times m$-dimensional.

We write the Nahm data in the left interval as

$$
T_{a}^{L}=\left(\begin{array}{c|c}
M_{a} & A_{a}^{\dagger} \\
\hline A_{a} & b_{a}
\end{array}\right)=\left(\begin{array}{cc|c}
N_{a} & E_{a}^{\dagger} & F_{a}^{\dagger} \\
E_{a} & P_{a} & G_{a}^{\dagger} \\
\hline F_{a} & G_{a} & b_{a}
\end{array}\right),
$$

where in the second equality we have separated the $(m+k-1)$-dimensional $M_{a}$ into blocks of dimension $m$ and $(k-1)$.

The same arguments as in the $\mathrm{SU}(2)$ case show that away from the boundary, the $A_{a}$ and $A_{a}^{\dagger}$ are negligible and the $M_{a}$ obey the $(m+k-1)$-monopole Nahm equations 5 However we must make sure that the $N_{a}$ are constant in the boundary region $(-1 / D, 0)$ as $D \rightarrow \infty$,

\footnotetext{
${ }^{5} \mathrm{As}$ in the $\mathrm{SU}(2)$ case, we can eliminate a constant from $A_{3}$ by a unitary transformation of the form of Eq. (3.4). We show in Appendix A that $A_{3}$ is at most of order $l$, so the shifts in the $M_{a}$ are negligible.
} 


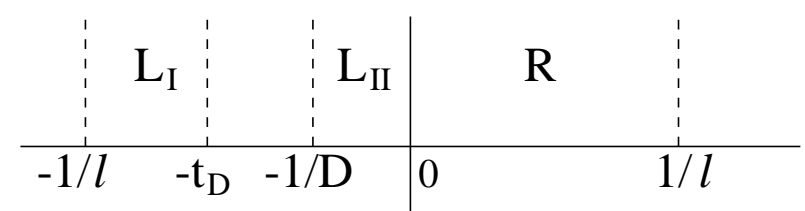

FIG. 1. The boundary region near $t=0$. We denote the interval $\left(-1 / l,-t_{D}\right)$ by $\mathrm{L}_{\mathrm{I}},(-1 / D, 0)$ by $\mathrm{L}_{\mathrm{II}}$, and $(0,1 / l)$ by $\mathrm{R}$.

so that they can continuously connect to the $T_{a}^{R}$ in the next interval. In Appendix A we show that the transition of the poles in the $T_{a}^{L}$ is similar to the $\mathrm{SU}(2)$ case. In terms of the regions shown in Fig.1, we have

$$
\begin{aligned}
\text { Region } \mathrm{L}_{\mathrm{I}}: & T_{1,2}^{L}(t)=\left(\begin{array}{cc}
T_{1,2, \text { red }}^{L} & \mathcal{O}(D) e^{-D|t|} \\
\mathcal{O}(D) e^{-D|t|} & \mathcal{O}(l)
\end{array}\right), \quad-1 / l<t<-t_{D}, \\
T_{3}^{L}(t) & =\left(\begin{array}{cc}
T_{3, \text { red }}^{L} & \mathcal{O}(l) e^{-D|t|} \\
\mathcal{O}(l) e^{-D|t|} & D+\mathcal{O}(l)
\end{array}\right), \quad-1 / l<t<-t_{D},
\end{aligned}
$$

$$
\text { Region } \mathrm{L}_{\mathrm{II}}: T_{a}^{L}(t)=T_{a, \text { orig }}^{L}, \quad-1 / D<t<0
$$

The discussion of the construction equation is similar to the $\mathrm{SU}(2)$ case and included in Appendix A.

\section{B. $k<0$ : Increasing the dimension of the pole term on the right}

We now remove a $(1,0)$ monopole from an $(m-|k|, m)$ configuration and see how a higher dimensional pole arises in $T_{a}^{R}$. In the original $(m-|k|, m)$ problem, $T_{a, \text { orig }}^{L}$ is $(m-$ $|k|) \times(m-|k|)$-dimensional. Near the boundary, $0<t<1 / D$,

$$
T_{a, \text { orig }}^{R}=\left(\begin{array}{cc}
N_{a}^{\prime} & \mathcal{O}\left(t^{(k-1) / 2}\right) \\
\mathcal{O}\left(t^{(k-1) / 2}\right) & -\frac{J_{a}^{(|k|)}}{t}
\end{array}\right),
$$

where the $N_{a}^{\prime}(0)=T_{a \text {,orig }}^{L}(0)$ are $(m-|k|) \times(m-|k|)$-dimensional. Here both $T_{3 \text {,orig }}^{L}$ and $N_{3}^{\prime}$ have one large eigenvalue $D$. We want to show that this problem is equivalent to the reduced $(m-|k|-1, m)$ problem where $T_{a \text {,red }}^{L}$ is $(m-|k|-1) \times(m-|k|-1)$-dimensional and $\mathcal{O}(l)$, while 


$$
T_{a, \text { red }}^{R}=\left(\begin{array}{cc}
N_{a} & \mathcal{O}\left(t^{k / 2}\right) \\
\mathcal{O}\left(t^{k / 2}\right) & -\frac{J_{a}^{(|k|+1)}}{t}
\end{array}\right),
$$

for $0<t<1 / l$, with $N_{a}(0)=T_{a, \text { red }}^{L}(0)$.

We decompose the Nahm data $T_{a}^{L}$ as in Eq. (3.3),

$$
T_{a}^{L}=\left(\begin{array}{cc}
M_{a} & A_{a}^{\dagger} \\
A_{a} & b_{a}
\end{array}\right)
$$

but with the $M_{a}$ being $(m-|k|-1)$-dimensional.

Because the off-diagonal terms $A_{a}$ must match on to the $N_{a}^{\prime}$, they can be at most of $\mathcal{O}(l)$ at $t=0$. This is different from the situation in Sec. IIII. Thus, in the large $D$ limit, the only divergent element of $T_{a}^{L}$ at the boundary is $b_{3}=D$. Since the $T_{a}^{L}$ are continuously connected to the $(m-|k|) \times(m-|k|)$ upper diagonal block of $T_{a}^{R}$, this requires that the corresponding element of $T_{a}^{R}$ also be divergent at $t_{2}$. The Nahm Eqs. (2.1) require this divergent behavior to be a simple pole and the residues to be $(|k|+1)$-dimensional $\mathrm{SU}(2)$ representations, which is irreducible due to the irreducibility of the residue in the original problem within $(0,1 / D)$.

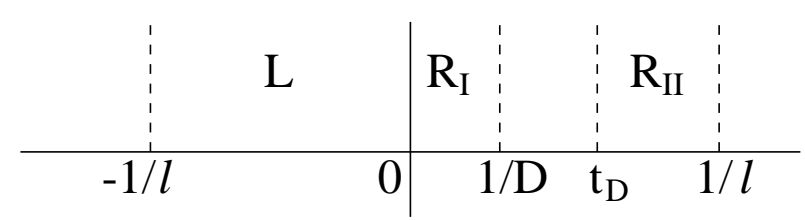

FIG. 2. The boundary region at $t=0$. We denote the interval $(-1 / l, 0)$ by $\mathrm{L},(0,1 / D)$ by $\mathrm{R}_{\mathrm{I}}$, and $\left(t_{D}, 1 / l\right)$ by $\mathrm{R}_{\mathrm{II}}$.

In terms of the regions shown in Fig. 2, the transition of the pole behavior is

$$
\text { Region L : } \quad T_{a}^{L}(t)=\left(\begin{array}{cc}
T_{a, \text { red }}^{L} & \mathcal{O}(l) e^{-D|t|} \\
\mathcal{O}(l) e^{-D|t|} & D \delta_{a 3}+\mathcal{O}(l)
\end{array}\right), \quad-1 / l<t<0,
$$

\footnotetext{
${ }^{6}$ The block-diagonal form in region L can be obtained by the unitary transformation Eq. (3.4), as before. We now also do a corresponding unitary transformation $\left(\begin{array}{cc}U & 0 \\ 0 & I_{|k| \times|k|}\end{array}\right)$ on the $T_{a}^{R}$, thus maintaining the boundary condition at $t=0$.
} 


$$
\begin{aligned}
& \text { Region } \mathrm{R}_{\mathrm{I}}: T_{a}^{R}(t)=T_{a, \text { orig }}^{R}, \quad 0<t<1 / D \\
& \text { Region } \mathrm{R}_{\mathrm{II}}: T_{a}^{R}(t)=T_{a, \text { red }}^{R}, \quad t_{D}<t<1 / l \text {. }
\end{aligned}
$$

As in the previous case, the construction equation on the left interval $\left(t_{1}, 0\right)$ has a solution $v_{+}$localized near $t=0$. On the right interval, there is a solution $v_{R}$ that behaves as $(\Delta t)^{-(|k|+2) / 2}$ in $\left(t_{D}, 1 / l\right)$ and that would have been discarded due to non-normalizability in the $(m-|k|-1, m)$ description, that becomes normalizable in $(0,1 / D)$ because the dimension of the pole residue has decreased. However, like $v_{+}$, this solution decays quickly away from the boundary. By reasoning similar to that at the end of Sec. [II, these solutions have no effect on the fields in the $D \rightarrow \infty$ limit. Thus the original $(m+k, m)$ description goes over to the reduced $(m+k-1, m)$ description.

\section{THE JUMPING DATA}

\section{A. $k=1$ : Emergence of the jumping data}

As described in the introduction, we want to investigate how the jumping data appear when a monopole is removed from a configuration that originally has no jumping data. We start with an $(m+1, m)$ configuration in the SU(3) theory. The Nahm data of interest for this system are three $(m+1) \times(m+1)$ Hermitian matrices $T_{a}^{L}$ defined on $\left(t_{1}, 0\right)$, and three $m \times m$ Hermitian matrices $T_{a}^{R}$ defined on $\left(0, t_{3}\right)$ that are continuously connected to the upper diagonal $m \times m$ block of the $T_{a}^{L}$. We write

$$
T_{a}^{L}=\left(\begin{array}{cc}
M_{a} & A_{a}^{\dagger} \\
A_{a} & b_{a}
\end{array}\right)
$$

where the $M_{a}$ are $m \times m$ dimensional, the $A_{a}^{\dagger}$ are $m$-dimensional vector and the $b_{a}$ are real. Again, we choose $b_{a}=\delta_{a 3} D+\mathcal{O}(l)$. The Nahm equations are decomposed as in Eqs. (3.6-3.12).

It is easier to analyze the Nahm equation when they are block-diagonalized in the middle of the interval. Using the same arguments as in Sec. [II, we first do a unitary transformation to subtract an additive constant from $A_{3}$. We will denote the matrices after this transformation with an extra prime. Two of the decomposed Nahm equation become

$$
\begin{aligned}
\frac{d M_{a}^{\prime}}{d t} & =i \epsilon_{a b c}\left(M_{b}^{\prime} M_{c}^{\prime}+A_{b}^{\prime \dagger} A_{c}^{\prime}\right), \\
\frac{d A_{3}^{\prime}}{d t} & =i \epsilon_{3 b c}\left(A_{b}^{\prime} M_{c}^{\prime}+B_{b}^{\prime} A_{c}^{\prime}\right),
\end{aligned}
$$


The exponential dependence of $A$ in Eq. (3.14) can now be extended all the way to the boundary $t=0$. This is because, unlike the $\mathrm{SU}(2)$ case in Sec. [II], we no longer have poles at the boundary. The $M_{a}$ are required to continuously connect to the matrices on the right interval and are always $\mathcal{O}(l)$. We then have

$$
A_{1,2}^{\prime}=\tilde{A}_{1,2} \sqrt{2 D} e^{-D|t|}
$$

where the $t$-independent $\tilde{A}_{1,2}$ are $\mathcal{O}(\sqrt{l})$ and related by

$$
\tilde{A}_{1}=i \tilde{A}_{2} .
$$

Notice that although we have the same exponential behavior as in Sec. III, the orders of magnitude are different. This is because, if the orders of magnitude of $\tilde{A}_{1,2}$ are bigger than those in Eq. (5.4), then by Eq. (5.2) they will cause the variation of $M_{a}^{\prime}$ around the boundary to exceed $\mathcal{O}(l)$. From Eq. (5.3) we get

$$
A_{3}^{\prime}=\mathcal{O}\left(l^{3 / 2} / D^{1 / 2}\right) .
$$

From Eq. (5.2) and the orders of magnitude in Eqs. (5.4) and (5.6), we can see that, for $t<-t_{D} / 2$, the $M_{a}^{\prime}$ satisfy the $m$-monopole Nahm equation.

However, the value of $A_{3}$ before the unitary transformation of Eq. (3.4) does not have to be as small as in Eq. (5.6), but can instead contain a constant term of $\mathcal{O}(\sqrt{l D})$. This general form of $T_{a}^{L}$ can be obtained by doing the inverse of the unitary transformation on $T_{a}^{\prime}$. This gives

$$
\begin{aligned}
A_{1,2} & =\tilde{A}_{1,2} \sqrt{2 D} e^{-D|t|}+\mathcal{O}\left(l^{3 / 2} / D^{1 / 2}\right) \\
A_{3} & =\tilde{A}_{3} \sqrt{D / 2}+\mathcal{O}\left(l^{3 / 2} / D^{1 / 2}\right)
\end{aligned}
$$

The shift caused by this unitary transformation is no longer negligible, as it was in Secs. III and IV. From Eq. (3.5), it is

$$
M_{3}=M_{3}^{\prime}+\frac{1}{2} \tilde{A}_{3}^{\dagger} \tilde{A}_{3}
$$

\footnotetext{
${ }^{7}$ The factor of $1 / 2$ arises because of the difference in the orders of magnitude of $\tilde{A}_{a}$ between here and Sec. III.

${ }^{8}$ The $A_{3}$ cannot be bigger than $\mathcal{O}(\sqrt{l D})$, since otherwise it would cause the $M_{a}$ to vary too much near the boundary $t=0$, as we will see from Eq. (5.10).
} 
From Eq. (5.2) and the forms of the $A_{a}$, we can see that within $\left(-t_{D} / 2,0\right)$ the $M_{a}$ are rapidly varying,

$$
M_{a}(0)-M_{a}\left(-t_{D} / 2\right)=-i \epsilon_{a b c} \tilde{A}_{b}^{\dagger} \tilde{A}_{c}
$$

In the following we will see that for the effective construction problem the discontinuity in the Nahm data at the boundary is

$$
M_{a}(0)-M_{a}^{\prime}\left(-t_{D} / 2\right)=-i \epsilon_{a b c} \tilde{A}_{b}^{\dagger} \tilde{A}_{c}+\frac{1}{2} \tilde{A}_{3}^{\dagger} \tilde{A}_{3} \delta_{a 3}
$$

where $M_{a}(0)=T_{a}^{R}(0)$ is the boundary value of the Nahm data $T_{a}^{R}$ at $t=0$. Because, as we will show, the rapid variation of $M_{a}^{\prime}$ in the infinitesimally small region $\left(-t_{D} / 2,0\right)$ has only negligible effects on the construction solutions in the large $D$ limit, $M_{a}^{\prime}\left(-t_{D} / 2\right)$ is effectively the boundary value of the left side Nahm data in the reduced problem.

To see this, we need to look at the solutions of the construction equation. As with the Nahm equations, it is easier to study the solutions using the block-diagonalized form of the Nahm data $T_{a}^{\prime}$ obtained by the unitary transformation. We decompose the corresponding construction solution as

$$
v^{\prime}=\left(\begin{array}{l}
w^{\prime} \\
z^{\prime}
\end{array}\right),
$$

where $w^{\prime}$ is a $2 m$-dimensional vector and $z^{\prime}$ is 2-dimensional. The decomposition of Eq. (2.2) is the same as in Eqs. (3.21) and (3.22):

$$
\begin{aligned}
& 0=-\frac{d}{d t} w^{\prime}+\left[\left(-M_{a}^{\prime}+r_{a}\right) \otimes \sigma_{a}\right] w^{\prime}-\left(A_{a}^{\prime \dagger} \otimes \sigma_{a}\right) z^{\prime}, \\
& 0=-\frac{d}{d t} z^{\prime}-\left(A_{a}^{\prime} \otimes \sigma_{a}\right) w^{\prime}+\left[\left(-b_{a}^{\prime}+r_{a}\right) \otimes \sigma_{a}\right] z^{\prime} .
\end{aligned}
$$

It is clear from Eqs. (5.4) and (5.6) that in the middle of the interval (i.e., $t<-t_{D} / 2$ ) $w^{\prime}$ and $z^{\prime}$ are decoupled. In the following we will consider two types of decoupled solutions. We will start in the middle of the interval and then study their behavior near the boundary.

For the first type, neglecting the terms which vanish as $D \rightarrow \infty$, we have only the $w^{\prime}$ components

$$
v^{\prime}=\left(\begin{array}{c}
w^{\prime} \\
0
\end{array}\right)
$$

Near the boundary region, the second term in Eq. (5.14) is $\mathcal{O}\left(w^{\prime} \sqrt{l D}\right) e^{-D|t|}$. After integrating across the boundary region, this gives the $z^{\prime}$ components a contribution of $\mathcal{O}\left(w^{\prime} \sqrt{l / D}\right)$, 
which is still negligible compared to $w^{\prime}$. Hence, the $w^{\prime}$ in Eq. (5.15) is a solution of Eq. (5.13) with the last term ignored. Furthermore, the rapid $\mathcal{O}(l) e^{-D|t|}$ variation of the $M_{a}^{\prime}$ is restricted to an interval of width $t_{D} / 2$, which is too small to significantly affect $w^{\prime}$. Hence the $w^{\prime}$ components in Eq. (5.15) solve the construction equation defined by the Nahm data $\hat{M}_{a}$ that are defined by $\hat{M}_{a}=M_{a}$ for $t<-t_{D} / 2$ and $\hat{M}_{a}=$ constant for $-t_{D} / 2<t \leq 0$. From this definition, we can see that at the boundary the $\hat{M}_{a}$ are not continuously connected to $T_{a}^{R}$, but instead have a jump given by Eq. (5.11).

In the second type of solution, the $w^{\prime}$ components are much smaller than the $z^{\prime}$ components. The latter can be obtained by ignoring the second term in Eq. (5.14). We have two such solutions, both similar to those in Eq. (3.25). We are interested in the one that is localized near $t=0$,

$$
z^{\prime}=S\left(\begin{array}{c}
0 \\
\sqrt{2 D} e^{-D|t|}
\end{array}\right)
$$

where $S$ is $t$-independent. To get the boundary behavior of the $w^{\prime}$ component, we plug this into Eq. (5.13) and obtain

$$
0=-\frac{d}{d t} w^{\prime}-\left(2 D e^{-2 D|t|}\right) S a^{\prime \dagger},
$$

where we assumed the large $D$ limit and defined

$$
a^{\prime \dagger}=\left(\begin{array}{c}
\tilde{A}_{1}^{\dagger}-i \tilde{A}_{2}^{\dagger} \\
0
\end{array}\right) .
$$

Thus, this zero-mode is

$$
v_{\text {jump }}^{\prime}=S\left(\begin{array}{c}
-a^{\prime \dagger} e^{-2 D|t|} \\
0 \\
\sqrt{2 D} e^{-D|t|}
\end{array}\right)
$$

In contrast with the localized solutions studied in the previous cases, the lower two components of $v_{\text {jump }}^{\prime}$ terminate at $t=0$ and need not satisfy any boundary conditions. Hence, in this case $v_{\text {jump }}^{\prime}$ gives a linearly independent solution that is concentrated within an interval of width $1 / D$ adjacent to the boundary. In the $D \rightarrow \infty$ limit, it is orthogonal to the other solutions and has norm $S^{\dagger} S$. Note that while the $w^{\prime}$ components are of order $\sqrt{l / D}$ smaller than $z^{\prime}$, they are $\mathcal{O}(l)$ at the boundary and cannot be neglected.

To get the general solution of the original problem, we must do an inverse unitary transformation 


$$
v^{\prime} \rightarrow v=\left[\left(\begin{array}{cc}
1 & A_{3}^{\dagger} / D \\
-A_{3} / D & 1
\end{array}\right) \otimes I_{2}\right] v^{\prime}
$$

on the solutions of Eqs. (5.15) and (5.19).

For the first type of solution, Eq. (5.15), $w=w^{\prime}$ up to terms that can be neglected. The $z^{\prime}$ remain small. Hence, in the normalization integral, Eq. (2.3), and the field integrals, Eq. (2.4), the only contributions come from the $w$ components, which satisfy the construction equation defined by the $m$-monopole Nahm data $\hat{M}_{a}$ defined below Eq. (5.15).

For the second type of solution, Eq. (5.19), the $w$ components receive contributions from the $z$ components after the transformation. We have

$$
v_{\text {jump }}=S\left(\begin{array}{c}
\left(-\tilde{A}_{1}^{\dagger}+i \tilde{A}_{2}^{\dagger}\right) e^{-2 D|t|} \\
\tilde{A}_{3}^{\dagger} e^{-D|t|} \\
0 \\
\sqrt{2 D} e^{-D|t|}
\end{array}\right) .
$$

At the boundary the upper components obey $w_{\text {jump }}(0)=-S a^{\dagger}$, where

$$
a^{\dagger}=\left(\begin{array}{c}
\tilde{A}_{1}^{\dagger}-i \tilde{A}_{2}^{\dagger} \\
-\tilde{A}_{3}^{\dagger}
\end{array}\right)=\tilde{A}_{a}^{\dagger} \otimes \sigma_{a}\left(\begin{array}{l}
0 \\
1
\end{array}\right)
$$

This satisfies

$$
\begin{aligned}
-\frac{1}{2} \operatorname{tr}_{2}\left(a^{\dagger} a \sigma_{a}\right) & =-\frac{1}{2}\left(i \epsilon_{a b c} \tilde{A}_{b}^{\dagger} \tilde{A}_{c}+\delta_{a 3} \tilde{A}_{b}^{\dagger} \tilde{A}_{b}-\tilde{A}_{a}^{\dagger} \tilde{A}_{3}-\tilde{A}_{3}^{\dagger} \tilde{A}_{a}\right) \\
& =-i \epsilon_{a b c} \tilde{A}_{b}^{\dagger} \tilde{A}_{c}+\frac{1}{2} \tilde{A}_{3}^{\dagger} \tilde{A}_{3} \delta_{a 3} \\
& =T_{a}^{R}(0)-\hat{M}_{a}(0) .
\end{aligned}
$$

Equation (5.5) has been used in the second equality, while Eq. (5.11) and the definition of the $\hat{M}_{a}$ have been used in the third equality. Comparing with Eq. (2.6), we see that the $a$ defined in Eq. (5.22) is the jumping data for the reduced problem. In the large $D$ limit, the rapid variation in the $w$ component of Eq. (5.21) over this interval gives

$$
\Delta w_{\text {jump }}=w_{\text {jump }}(0)-w_{\text {jump }}\left(-t_{D} / 2\right) \approx w_{\text {jump }}(0)=-S a^{\dagger}
$$

This is just the discontinuity expected from Eq. (2.7). As noted above, the inner products containing $v_{\text {jump }}$ are dominated by the $z$ components. These produce the second terms on the right hand sides of Eqs. (2.8) and (2.9). 
(In the above discussion, we have mainly concentrated on the region around the middle boundary $t=0$. While boundary conditions at other boundaries may eliminate some of the other solutions, they cannot eliminate $v_{\text {jump }}$, because it is localized at $t=0$.)

A general solution of the construction equation is a linear combination of these two types of solutions. In the $D \rightarrow \infty$ limit, the contribution of the first type to the upper components is continuous, while that from the second type has a discontinuity of the form of Eq. (5.24). Only the second type of solution has nonzero lower components, and their only effects are to give contributions to the normalization and field integrals that are quadratic in $S$.

Thus, we have two different but equivalent ways of looking at this problem. From the point of view of the original $(m+1, m)$ ADHMN construction with one monopole far away, the Nahm data $T_{a}^{L}$ gives two types of solutions: those which have negligible lower components, and the localized solution of Eq. (5.21). From the point of view of the reduced $(m, m)$ construction, the upper components of the first type of solution satisfy the construction equation given by the reduced Nahm data $\hat{M}_{a}$ and may be discontinuous at the boundary. The effect of the localized solution is replaced by the jumping data that describe the discontinuity of the Nahm data $\hat{M}_{a}$.

In the reduced problem, the jumping data is part of the Nahm data. It is interesting to note that, although the jumping data arise from a localized solution of the construction equation of the original problem, they are given, through Eq. (5.22), by the off-diagonal elements $A_{a}$ of the original Nahm matrices.

\section{B. $k=0:$ Disappearance of the jumping data}

In this subsection, we start with an $(m, m)$ monopole configuration that has jumping data. We again consider the limit where one of the $(1,0)$ monopoles is displaced by a distance $D \gg l$ along the $z$-axis. Using the same arguments and notation as in Sec. $\nabla \mathrm{A}$, we find that the original Nahm data $T_{a}^{L}$ generically take the form

$$
\begin{aligned}
T_{1,2}^{L} & =\left(\begin{array}{cc}
\mathcal{O}(l) & \tilde{A}_{1,2}^{\dagger} \sqrt{2 D} e^{-D|t|}+\mathcal{O}\left(l^{3 / 2} / D^{1 / 2}\right) \\
\tilde{A}_{1,2} \sqrt{2 D} e^{-D|t|}+\mathcal{O}\left(l^{3 / 2} / D^{1 / 2}\right) & \mathcal{O}(l)
\end{array}\right), \\
T_{3}^{L} & =\left(\begin{array}{cc}
\mathcal{O}(l) & \tilde{A}_{3}^{\dagger} \sqrt{D / 2}+\mathcal{O}\left(l^{3 / 2} / D^{1 / 2}\right) \\
\tilde{A}_{3} \sqrt{D / 2}+\mathcal{O}\left(l^{3 / 2} / D^{1 / 2}\right) & D+\mathcal{O}(l)
\end{array}\right) .
\end{aligned}
$$

Evaluating this at the boundary $t=0$, we get 


$$
\begin{gathered}
T_{1,2}^{L}(t=0)=\left(\begin{array}{cc}
\mathcal{O}(l) & \tilde{A}_{1,2}^{\dagger} \sqrt{2 D}+\mathcal{O}\left(l^{3 / 2} / D^{1 / 2}\right) \\
\tilde{A}_{1,2} \sqrt{2 D}+\mathcal{O}\left(l^{3 / 2} / D^{1 / 2}\right) & \mathcal{O}(l)
\end{array}\right), \\
T_{3}^{L}(t=0)=\left(\begin{array}{cc}
\mathcal{O}(l) & \tilde{A}_{3}^{\dagger} \sqrt{D / 2}+\mathcal{O}\left(l^{3 / 2} / D^{1 / 2}\right) \\
\tilde{A}_{3} \sqrt{D / 2}+\mathcal{O}\left(l^{3 / 2} / D^{1 / 2}\right) & D+\mathcal{O}(l)
\end{array}\right),
\end{gathered}
$$

where the $\tilde{A}_{a}$ are $\mathcal{O}(\sqrt{l})$. We write the jumping data as

$$
a=\left(\begin{array}{ll}
\alpha_{1} & \beta_{1} \\
\alpha_{2} & \beta_{2}
\end{array}\right),
$$

where 1,2 are spinor indices, $\alpha_{1,2}$ are $m$-1-dimensional row vectors and $\beta_{1,2}$ are complex numbers. In this notation, the discontinuities of the Nahm data, Eq. (2.6), are

$$
\begin{aligned}
& \Delta T_{1}=-\frac{1}{2}\left(\begin{array}{cc}
\alpha_{1}^{\dagger} \alpha_{2}+\alpha_{2}^{\dagger} \alpha_{1} & \alpha_{1}^{\dagger} \beta_{2}+\alpha_{2}^{\dagger} \beta_{1} \\
\beta_{1}^{\dagger} \alpha_{2}+\beta_{2}^{\dagger} \alpha_{1} & \beta_{1}^{\dagger} \beta_{2}+\beta_{2}^{\dagger} \beta_{1}
\end{array}\right), \\
& \Delta T_{2}=\frac{i}{2}\left(\begin{array}{cc}
\alpha_{1}^{\dagger} \alpha_{2}-\alpha_{2}^{\dagger} \alpha_{1} & \alpha_{1}^{\dagger} \beta_{2}-\alpha_{2}^{\dagger} \beta_{1} \\
\beta_{1}^{\dagger} \alpha_{2}-\beta_{2}^{\dagger} \alpha_{1} & \beta_{1}^{\dagger} \beta_{2}-\beta_{2}^{\dagger} \beta_{1}
\end{array}\right), \\
& \Delta T_{3}=-\frac{1}{2}\left(\begin{array}{cc}
\alpha_{1}^{\dagger} \alpha_{1}-\alpha_{2}^{\dagger} \alpha_{2} & \alpha_{1}^{\dagger} \beta_{1}-\alpha_{2}^{\dagger} \beta_{2} \\
\beta_{1}^{\dagger} \alpha_{1}-\beta_{2}^{\dagger} \alpha_{2} & \beta_{1}^{\dagger} \beta_{1}-\beta_{2}^{\dagger} \beta_{2}
\end{array}\right) .
\end{aligned}
$$

Since all elements of the $T_{a}^{R}$ are $\mathcal{O}(l)$ for $t \geq 0$, we have

$$
T_{a}^{L}(t=0)+\Delta T_{a}=\mathcal{O}(l) .
$$

By comparing the $m m$-elements of Eqs. (5.25) and (5.27), we get

$$
\beta_{1}=\sqrt{2 D}+\mathcal{O}(l / \sqrt{D}), \quad \beta_{2}=\mathcal{O}(l / \sqrt{D}),
$$

where an arbitrary phase can be absorbed in the redefinition. Using the constraint from Eq. (5.5), we find from the off-diagonal blocks in Eqs. (5.25) and (5.27) that

$$
\alpha_{1}=\tilde{A}_{3}+\mathcal{O}\left(l^{3 / 2} / D\right), \quad \alpha_{2}=2 \tilde{A}_{1}+\mathcal{O}\left(l^{3 / 2} / D\right) .
$$

These results imply that the discontinuities in the upper-left $(m-1) \times(m-1)$ matrices in Eq. (5.27) can be written as 


$$
\Delta M_{a}=i \epsilon_{a b c} \tilde{A}_{b}^{\dagger} \tilde{A}_{c}-\frac{1}{2} \tilde{A}_{3}^{\dagger} \tilde{A}_{3} \delta_{a 3}+\mathcal{O}\left(l^{2} / D\right) .
$$

As before, by means of a unitary transformation we can make the $T_{a}^{L}$ block diagonal away from the boundary and then define $(m-1) \times(m-1)$ Nahm data $\hat{M}_{a}$. The effective difference between the $\hat{M}_{a}$ and the $M_{a}$ at the boundary is exactly -1 times the quantity in Eq. (5.31), as we saw in Eq. (5.11). Thus, by using the $\hat{M}_{a}$ and the $T_{a}^{R}$, the jumping data effectively disappear in the large $D$ limit.

As in Sec. VA, all but one of the solutions of the construction equation have upper components $w$ that solve the construction equation associated with $\hat{M}_{a}$ and lower components $z$ that are negligible. The remaining solution is localized near $t=0$ and is of the form

$$
N\left(\begin{array}{c}
\mathcal{O}(\sqrt{l}) e^{-D|t|} \\
0 \\
\sqrt{2 D} e^{-D|t|}
\end{array}\right),
$$

where $N$ is constant and we have only indicated the order of the magnitude of the first $2 m-2$ components. From the jumping data in Eqs. (5.29) and (5.30), we see that the discontinuities in the construction equation solutions at $t=0$ must be of the form

$$
S\left(\begin{array}{c}
\mathcal{O}(\sqrt{l}) \\
\sqrt{2 D} \\
0
\end{array}\right),
$$

where the notation is the same as in Eq. (5.32). In order to connect properly to the solutions on the right interval, both $N$ and $S$ have to be proportional to $1 / \sqrt{D}$. Hence, neither the localized solution nor the jumping data contribute to the normalization or field integrals in the $D \rightarrow \infty$ limit. Furthermore, in this limit the upper $2 m-2$ components of the solutions become continuous at the boundary. Thus, the fields become the same as in the $(m-1, m)$ construction.

\section{AN EXPLICIT SU(3) EXAMPLE}

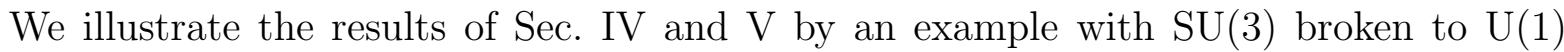
$\times \mathrm{U}(1)$ with asymptotic Higgs field $\Phi=\operatorname{diag}\left(t_{1}, t_{2}, t_{3}\right)$. We consider the $(2,1)$ monopole solution, whose explicit Nahm data are available. This example has been discussed in detail in [15]. Here we will be interested in the limit where one fundamental $(1,0)$ monopole is removed. We will choose the coordinates so that one $(1,0)$ monopole is at the origin and the other $(1,0)$ monopole is a distance $D$ away on the $z$-axis. The $(0,1)$ monopole is at a distance of order $l$ from the origin. 
The Nahm data in the right interval $\left(t_{2}, t_{3}\right)$ are simply real numbers that give the coordinates of the $(0,1)$ fundamental monopole. The boundary condition requires these constants to be equal to the boundary values of the 11-elements of the $2 \times 2$-dimensional Nahm data defined in the left interval $\left(t_{1}, t_{2}\right)$. If all three monopoles are collinear, rotations and translations the Nahm data in $\left(t_{1}, t_{2}\right)$ are [16]

$$
T_{a}^{L}=\frac{1}{2} f_{a} \sigma_{a}+\frac{D}{2} \delta_{a 3} \quad(\text { no sum })
$$

for $a=1,2,3$, where the $\sigma_{a}$ are Pauli matrices. The analytic functions $f_{a}$ are

$$
\begin{aligned}
& f_{1}(t)=-\frac{D c n_{k}\left(D\left(t-t_{1}\right)\right)}{s n_{k}\left(D\left(t-t_{1}\right)\right)}, \\
& f_{2}(t)=-\frac{D d n_{k}\left(D\left(t-t_{1}\right)\right)}{s n_{k}\left(D\left(t-t_{1}\right)\right)}, \\
& f_{3}(t)=-\frac{D}{s n_{k}\left(D\left(t-t_{1}\right)\right)},
\end{aligned}
$$

where $0 \leq k \leq 1$ and $D<\frac{2}{t_{2}-t_{1}} K(k)$. Here $s n_{k}, c n_{k}$ and $d n_{k}$ are the Jacobi elliptic functions and $K(k)$ is the complete elliptic integral of the first kind. The $f_{a}$ have poles at $t_{1}$ and $t_{*}=t_{1}+2 K(k) / D>t_{2}$.

In general, the relations between the parameters $k$ and $D$ and the physical quantities are complicated. They become simple when $k \rightarrow 1$ in the large $D$ limit. Within the pole region $t-t_{1} \ll 1 / D$, we have

$$
T_{a}^{L}=\frac{\sigma_{a}}{2\left(t-t_{1}\right)}+\mathcal{O}\left(D^{2}\left(t-t_{1}\right)\right) .
$$

Away from this region, $t-t_{1} \gg 1 / D$, we can use the approximation

$$
f_{1}=f_{2}=-D / \sinh \left(D\left(t-t_{1}\right)\right), \quad f_{3}=-D \operatorname{coth}\left(D\left(t-t_{1}\right)\right),
$$

valid when $k \rightarrow 1$, to obtain

$$
T_{1}^{L}=\left(\begin{array}{cc}
0 & -D e^{-D\left|t-t_{1}\right|} \\
-D e^{-D\left|t-t_{1}\right|} & 0
\end{array}\right),
$$

\footnotetext{
${ }^{9}$ If they are not collinear, we must do a unitary transformation on $T_{a}^{L}$, as we will see later in this section.
} 


$$
\begin{aligned}
& T_{2}^{L}=\left(\begin{array}{cc}
0 & i D e^{-D\left|t-t_{1}\right|} \\
-i D e^{-D\left|t-t_{1}\right|} & 0
\end{array}\right), \\
& T_{3}^{L}=\left(\begin{array}{cc}
-D e^{-2 D\left|t-t_{1}\right|} & 0 \\
0 & D+D e^{-2 D\left|t-t_{1}\right|}
\end{array}\right),
\end{aligned}
$$

for $t<\left(t_{1}+t_{*}\right) / 2$. (Higher order exponential terms have been omitted here, and in later similar expressions.) This explicitly verifies the predictions of Eqs. (3.17)-(3.19).

The expressions in the region $t>\left(t_{1}+t_{*}\right) / 2$ can be related to those in $t<\left(t_{1}+t_{*}\right) / 2$ by

$$
\begin{aligned}
& s n_{k}(x)=s n_{k}(2 K(k)-x), \\
& d n_{k}(x)=d n_{k}(2 K(k)-x), \\
& c n_{k}(x)=-c n_{k}(2 K(k)-x) .
\end{aligned}
$$

In particular, the upper-left element of $T_{3}^{L}$ has to be $\mathcal{O}(l)$ at $t=t_{2}$. Then we have

$$
\begin{aligned}
T_{1}^{L}=\left(\begin{array}{cc}
0 & D e^{-D\left|t-t_{*}\right|} \\
D e^{-D\left|t-t_{*}\right|} & 0
\end{array}\right) & =\left(\begin{array}{cc}
0 & \sqrt{D c} e^{-D\left|t-t_{2}\right|} \\
\sqrt{D c} e^{-D\left|t-t_{2}\right|} & 0
\end{array}\right), \\
T_{2}^{L}=\left(\begin{array}{cc}
0 & i D e^{-D\left|t-t_{*}\right|} \\
-i D e^{-D\left|t-t_{*}\right|} & 0
\end{array}\right) & =\left(\begin{array}{cc}
0 & i \sqrt{D c} e^{-D\left|t-t_{2}\right|} \\
-i \sqrt{D c} e^{-D\left|t-t_{2}\right|} & 0
\end{array}\right), \\
T_{3}^{L}=\left(\begin{array}{cc}
-D e^{-2 D\left|t-t_{*}\right|} & 0 \\
0 & D+D e^{-2 D\left|t-t_{*}\right|}
\end{array}\right) & =\left(\begin{array}{cc}
-c e^{-2 D\left|t-t_{2}\right|} & 0 \\
0 & D+c e^{-2 D\left|t-t_{2}\right|}
\end{array}\right),
\end{aligned}
$$

for $t>\left(t_{1}+t_{*}\right) / 2$, where $c=D e^{-2 D\left|t_{*}-t_{2}\right|}$ is a positive constant of $\mathcal{O}(l)$ that gives the position of the $(0,1)$ monopole at $(0,0,-c)$. These are examples of Eqs. (5.4) and (5.6).

Away from the boundaries, the Nahm data is approximately diagonal, with

$$
T_{1}^{L}=T_{2}^{L}=0, \quad T_{3}^{L}=\left(\begin{array}{ll}
0 & 0 \\
0 & D
\end{array}\right) .
$$

This corresponds to two widely separated $(1,0)$ monopoles, with one at the origin and the other at $D$ on the $z$-axis.

Eq. (5.14) gives two solutions localized near a boundary, with lower components 


$$
z_{-}=\sqrt{2 D}\left(\begin{array}{l}
1 \\
0
\end{array}\right) e^{-D\left|t-t_{1}\right|}, \quad z_{+}=\sqrt{2 D}\left(\begin{array}{l}
0 \\
1
\end{array}\right) e^{-D\left|t-t_{2}\right|} .
$$

The second one is localized near $t_{2}$. Substituting this into Eq. (5.13) gives

$$
w=\left(\begin{array}{c}
-\sqrt{2 c} e^{-2 D\left|t-t_{2}\right|} \\
0
\end{array}\right) .
$$

Although this has a nonzero value only at the boundary in the $D \rightarrow \infty$ limit, it provides an extra degree of freedom for connecting the solutions from the two intervals. Evaluating Eq. (6.10) at the boundary $t_{2}$ gives the jumping data $a^{\dagger}$, with the correct jump $\Delta T_{a}=$ $-\frac{1}{2} t r_{2}\left(a^{\dagger} a \sigma_{a}\right)=-c \delta_{a 3}$.

So far we have restricted ourselves to the case where all three monopoles are collinear. We now relax this restriction. Without loss of generality, we can rotate the system so that the $(1,0)$ monopoles are on the $z$-axis while the $(0,1)$ monopole is in the $x z$ plane at $(x, 0, z)$. To obtain the Nahm data for this solution, we perform a unitary transformation using

$$
\mathcal{U}=\left(\begin{array}{cc}
\cos \frac{\theta}{2} & \sin \frac{\theta}{2} \\
-\sin \frac{\theta}{2} & \cos \frac{\theta}{2}
\end{array}\right)
$$

on the previous Nahm data, with

$$
\sin \theta=x / \sqrt{D c}, \quad \text { where } c=\left(\sqrt{z^{2}+x^{2}}-z\right) / 2 \equiv(r-z) / 2 .
$$

This unitary transformation rotates the 11-elements at $t_{2}$ (i.e. the position of the $(0,1)$ monopole) along an ellipsoid with foci $(0,0,0)$ and $(0,0, D)$ on the $z$-axis [17]. The Nahm data after the transformation are (in the $D \rightarrow \infty$ limit)

$$
\begin{aligned}
& T_{1}^{L}=\left(\begin{array}{cc}
x e^{-D\left|t-t_{2}\right|} & \sqrt{\frac{D(r-z)}{2}} e^{-D\left|t-t_{2}\right|} \\
\sqrt{\frac{D(r-z)}{2}} e^{-D\left|t-t_{2}\right|} & -x e^{-D\left|t-t_{2}\right|}
\end{array}\right), \\
& T_{2}^{L}=\left(\begin{array}{cc}
0 & i \sqrt{\frac{D(r-z)}{2}} e^{-D\left|t-t_{2}\right|} \\
-i \sqrt{\frac{D(r-z)}{2}} e^{-D\left|t-t_{2}\right|} & 0
\end{array}\right),
\end{aligned}
$$




$$
T_{3}^{L}=\left(\begin{array}{cc}
\frac{x^{2}}{2(r-z)}-\frac{r-z}{2} e^{-2 D\left|t-t_{2}\right|} & \sqrt{\frac{D}{2}} \frac{x}{\sqrt{r-z}} \\
\sqrt{\frac{D}{2} \frac{x}{\sqrt{r-z}}} & D-\frac{x^{2}}{2(r-z)}+\frac{r-z}{2} e^{-2 D\left|t-t_{2}\right|}
\end{array}\right) .
$$

These are examples of Eqs. (5.7) and (5.8). This transformation changes Eq. (6.10) to

$$
\left(\begin{array}{c}
-\sqrt{r-z} e^{-2 D\left|t-t_{2}\right|} \\
\frac{x}{\sqrt{r-z}} e^{-D\left|t-t_{2}\right|}
\end{array}\right),
$$

again giving the correct jumps $\Delta T_{1}=x, \Delta T_{2}=0, \Delta T_{3}=z$.

\section{THE D-BRANE PICTURE}

As mentioned in the introduction, in the D-brane picture the monopoles are D-strings stretching between adjacent D3-branes. From the perspective of the D3-brane, the endpoints of the D-strings are magnetic sources that generate magnetic flux in the three spatial directions on the D3-brane.

We denote the spatial distance on the D3-brane as $r$ and the Higgs field as $\Phi$. The Higgs field of the D3-brane describes its transverse fluctuations (which we denote as $\hat{t}$ ) up to a factor of $2 \pi l_{s}^{2}$ (where $l_{s}$ is the string scale). The nontrivial profiles of the Higgs fields of the monopoles describe the bending of the D3-brane due to the presence of the D-strings [10,11. If we consider the case where the D-strings end at the D3-brane, one typically finds that spikes are created at the D-string endpoints. These spikes can be obtained from the asymptotic Higgs profile of the charge $k$ solution

$$
|\hat{t}|=2 \pi l_{s}^{2}|t|=2 \pi l_{s}^{2} \Phi(r)=\pi l_{s}^{2} \frac{|k|}{e r} .
$$

(For this section, we have restored the factors of $e$, with $r_{a} \rightarrow e r_{a}, T_{a} \rightarrow e T_{a}$.) This formula is valid if $r$ is bigger than the monopole separation scale $l$ and the monopole core size.

On the other hand, there is a dual description of the above phenomena from the perspective of the D-strings [18. As we have seen in the ADHMN construction, the $k \neq 0$ case corresponds to situations where the Nahm data on the two sides of the boundary have different dimensions. In this case (for $|k|>1$ ), we have poles emerging at the boundary. Since the Nahm data give the transverse fluctuations of the D-strings, these poles, with $k$-dimensional irreducible $\mathrm{SU}(2)$-valued residues, means that these D-strings are no longer distinct from each other near the D3-branes. They form a noncommutative two-sphere [19] 
and have an overall funnel-like geometry. The radius of this two-sphere is naturally defined as

$$
R(\hat{t})^{2}=\frac{4 \pi^{2} l_{s}^{4}}{|k|} \sum_{i=1}^{3} \operatorname{Tr}\left[T^{i}(\hat{t})^{2}\right] .
$$

In the pole region $|\hat{t}|<2 \pi l_{s}^{2} / e l$ we have

$$
R(\hat{t})=\pi l_{s}^{2} \frac{|k|}{e|\hat{t}|} \sqrt{1-\frac{1}{k^{2}}},
$$

where $\sum_{i}\left(J^{i}\right)^{2}=\frac{1}{4}\left(k^{2}-1\right) I_{k}$ has been used.

Noticing that the three transverse directions of the D-strings are the same as the three spatial directions on the D3-brane, we can then identify $R$ in Eq. (7.3) with $r$ in Eq. (7.1). These two dual descriptions, Eq. (7.1) and Eq. (7.3), of the brane junction agree well for large $k$. This can be explained as follows [18. This junction can be described by the nonAbelian world-volume Born-Infeld actions of the D3-branes and D-strings. This will give, respectively, the BPS monopole equation on the D3-branes and the Nahm equation on the D-string. The regions of validity of these two descriptions are restricted to the region where the effect of a derivative on the fields is less than a factor of $1 / l_{s}$, so that the higher order string corrections to the Born-Infeld actions can be ignored. This means $r \gg l_{s}$ in Eq. (7.1) and $|\hat{t}| \gg l_{s}$ in Eq. (7.3). These regions overlap when $|k| \gg 1$. In terms of $\hat{t}$, the overlapping region is $l_{s} \ll|\hat{t}| \ll k \pi l_{s} / e$. This overlapping region extends into the pole region of the Nahm data if $e<2 \pi l_{s} / l$. This is consistent with the weak electric coupling limit where the monopole description makes sense.

Now the transitions of the poles in Sec. IV can be interpreted in this D-brane picture. For the $k>1$ case, we only see a $k-1$ net magnetic charge in the spatial region $l \ll r \ll D$ on the D3-brane. In the dual description on the D-strings, this corresponds to the $(k-1)$ dimensional poles in the boundary region $|t| \ll 1 / e l$. If we move far away, to the spatial region $r \gg D$, we start to see the charge of the removed monopole. In the dual description, this corresponds to the higher dimensional poles within the boundary region $|t| \ll 1 / e D$. The $k<0$ case is similar, except now the removed monopole cancels one unit of magnetic charge. These two cases are sketched in Fig. 3 .

We also notice that in these two cases the distant D-string segment interacts predominantly with the noncommutative two-sphere part of the D-strings that contribute to the net magnetic charge on the D3-brane. These string excitations are described by the poles in the Nahm data. The interactions between this distant D-string segment and the other D-strings are small. For the $k>1$ case, these are described by the $F_{a}$ in Appendix A. These fall as 


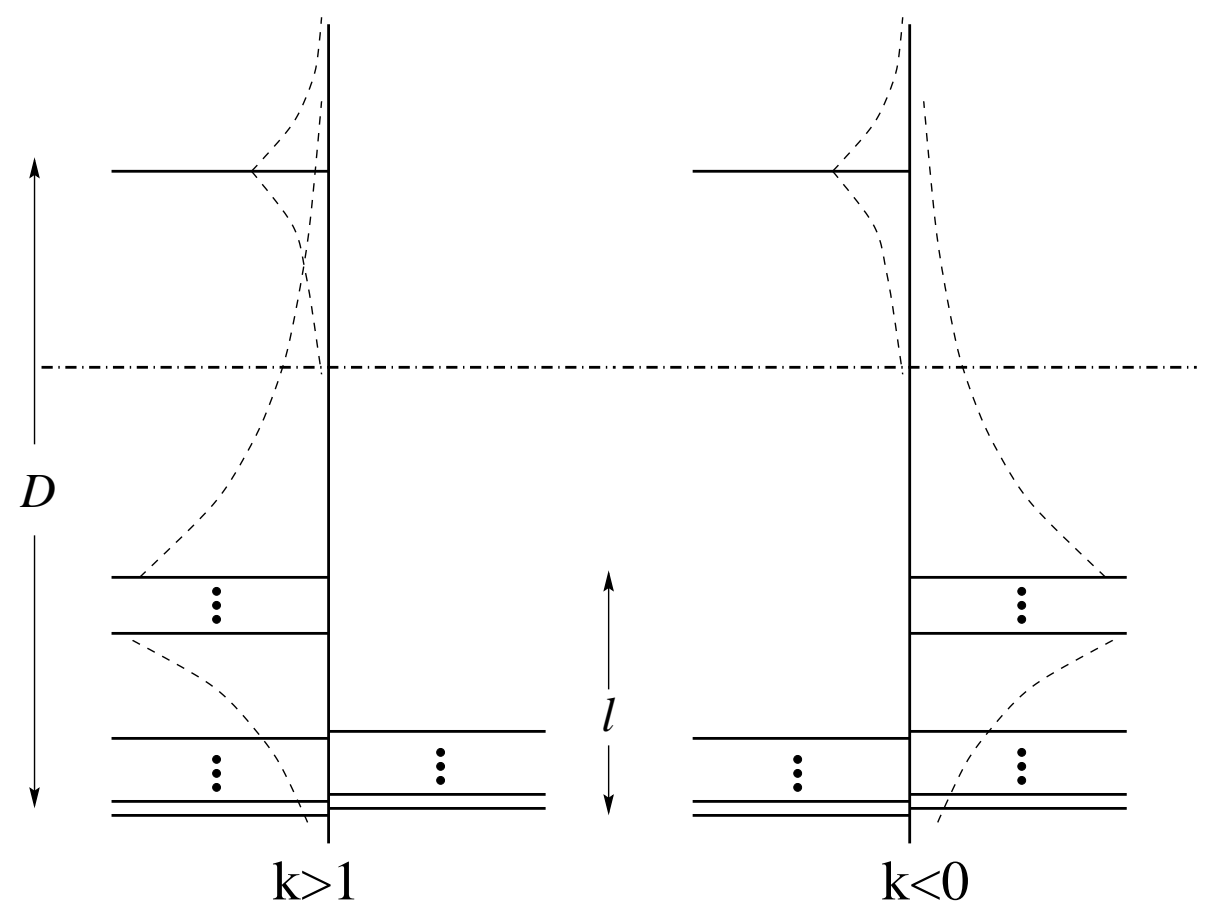

FIG. 3. The D-brane interpretation of the $k>1$ and $k<0$ cases. In this and the next two figures the perpendicular solid lines represent the D3-branes and the horizontal solid lines are the D-strings. The dashed-dotted lines separate the region of size $l$ from that of size $D$. In this figure the dashed lines represent the D3-brane spikes.

a power of $t$ and vanish at the boundary $t=0$. For the $k<0$ case, the interactions are described by the $A_{a}$ in Sec. IVB, which are at most of order $l$ at the boundary $t=0$.

The ADHMN construction with jumping data can be obtained by T-duality of the D0D4 system. One finds that the jumping data describe excitations of bosonic strings that stretch between the D-strings and D3-branes. These excitations are always localized on the D3-branes. This method naturally imposes the restriction that the number of D-string segments should be the same on both sides of each D3-brane. In other words, from the D3-brane point of view, all the magnetic flux coming from the D-strings on one side has to exit within a finite distance to the D-strings on the other side. We have seen that different boundary conditions can be naturally linked to the others by removing certain D-string segments. Therefore we can start with this configuration and derive all the other boundary conditions by removing certain D-string segments. It is then interesting to see how the appearance or the disappearance of the jumping data can be interpreted in this D-brane picture.

In addition, because of the T-duality, the system is compactified along the D1 direction. 


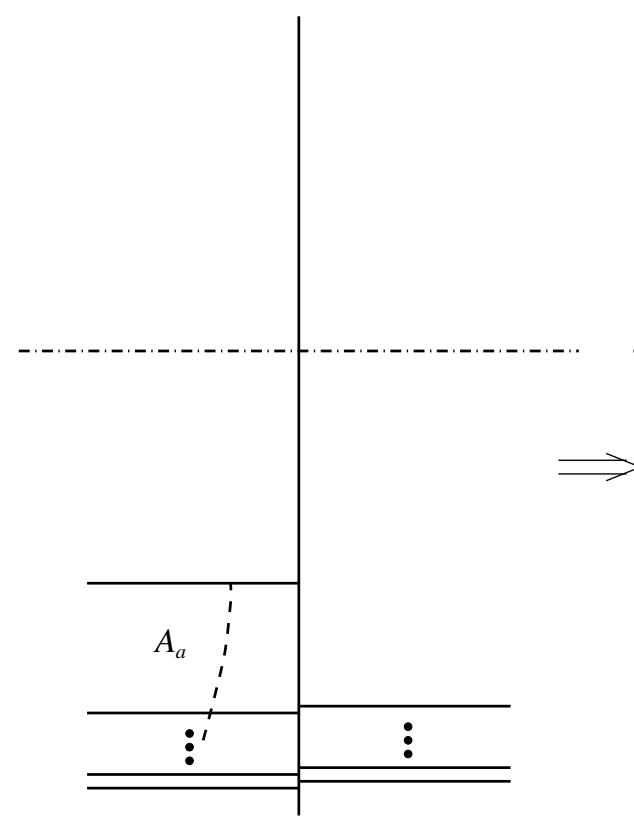

(a)

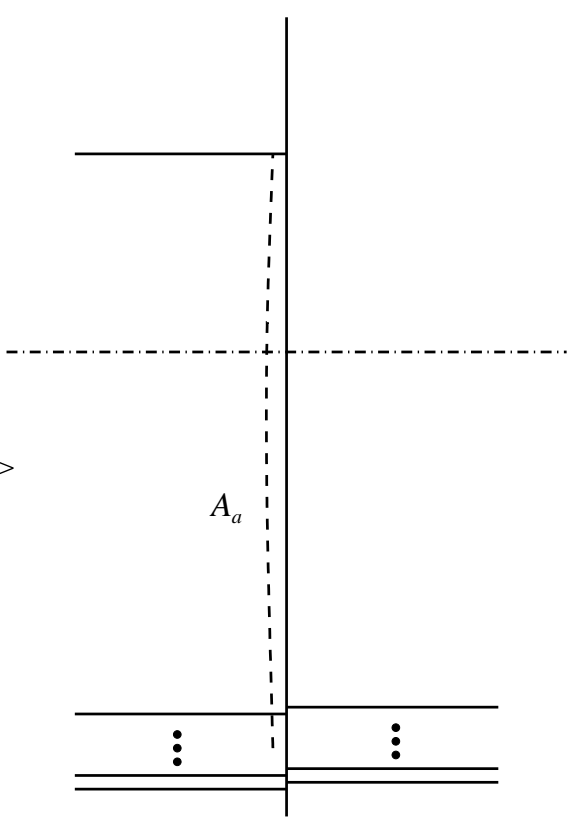

(b)

$\mathrm{k}=1$

FIG. 4. The D-brane interpretation of the $k=1$ case. In this figure the dashed lines represent the fundamental string excitations between the D-strings; (a) and (b) represent the D-brane pictures before and after removing a D-string segment to a distance $D$, respectively.

If we remove away all the D-string segments in one interval, we effectively decompactify the system.

We first look at the $k=1$ case. We focus on the fields describing the fundamental strings stretching between the D-string segment we want to separate and the rest of the D-strings. In the Nahm data, these fields are the $A_{a}$ of Sec. VA. Before the separation, they are within a region of size $1 / \mathrm{el}$ (in terms of the D-string coordinate $t$ ) adjacent to the D3-brane. As we remove the D-string segment to a distance of order $D$ (in terms of the distance on the D3brane), these fields become localized closer to the D3-brane; they are effectively restricted to a region of order $1 / e D$ as indicated by the $e^{-D|t|}$ dependence of the $A_{a}$ in Eqs. (5.7) and (5.8). Therefore, as $D \rightarrow \infty$ these string excitations will be restricted to the D3 brane and effectively appear as interactions between the D-strings and the D3-brane, just like the hypermultiplet. Quantitatively, as we can see from Eq. (5.22), the effective jumping data is indeed proportional to the $A_{a}$, with a normalization factor of order $\sqrt{D}$. Notice that, in contrast with the previous two cases, these string excitations are not small, even though the system left is neutral. They are of order $\sqrt{l D}$ at $t=0$. This case is sketched in Fig. 6 .

The $k=0$ case is a bit more complicated. Just as in the above $k=1$ case, as we remove 


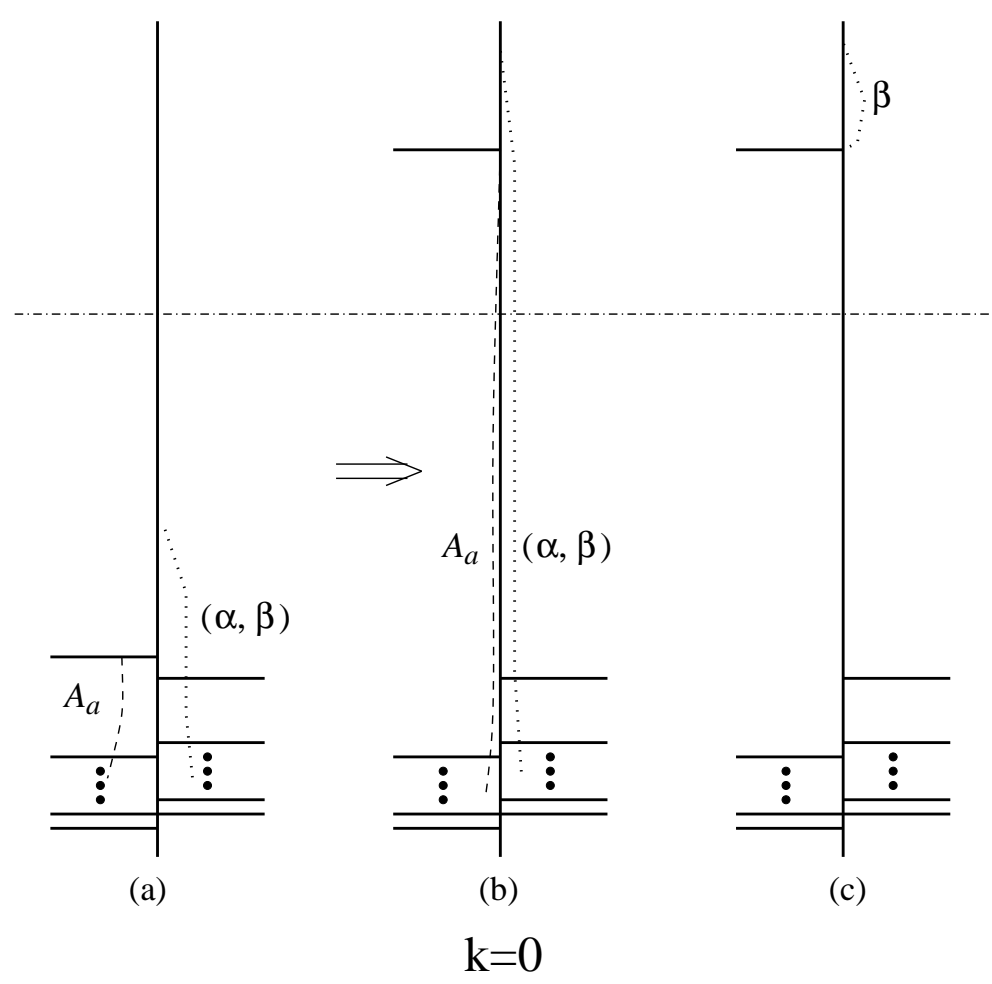

FIG. 5. The D-brane picture of the $k=0$ case. In this figure the dashed lines represent the fundamental string excitations between the D-strings; the dotted line is the hypermultiplet (jumping data). (a) represents the D-brane picture before we removing a D-string segment to a distance $D$. We use (b) and (c) to represent the D-brane picture after this separation, where (c) is the final effective picture.

one D-string segment the fundamental string modes connecting this D-string segment and the other D-strings become restricted to the D3 brane (see (b) in Fig. 5). One the other hand, we also have the jumping data between the D-string and D3 branes (see (b) in Fig. 5). Equation (5.28) requires that the leading, $\mathcal{O}(\sqrt{l D})$, parts of these two contributions cancel. This leaves the $\beta$ part of the jumping data, represented by (c) in Fig. 5. This decouples in the $D \rightarrow \infty$ limit.

\section{CONCLUSIONS AND DISCUSSION}

In this paper, we have related the boundary conditions in the ADHMN constructions for different magnetic charges by removing fundamental monopoles one by one to spatial infinity; we were particularly interested in the cases involving jumping data, which seem quite different from the other cases. We demonstrated the equivalence in the $D \rightarrow \infty$ limit between the ADHMN construction for the original $(\ldots, m+k, m, \ldots)$ problem, with one 
$(\ldots, 1,0, \ldots)$ fundamental monopole removed a distance $D$ from the others, and the reduced $(\ldots, m+k-1, m, \ldots)$ problem.

For the Nahm data $T_{a}^{L}$ on the left interval, we generally find a small region near the boundary whose width $\epsilon_{D}$ goes to zero as $D \rightarrow \infty$. Away from this boundary region, we can make the $T_{a}^{L}$ block-diagonal with $D \delta_{a 3}$ in one block and the decoupled Nahm data of the reduced problem in the other. The values of the $T_{a}^{L}$ at $t=-\epsilon_{D}$ become the boundary values for the reduced problem. The Nahm data generally vary rapidly in $-\epsilon_{D}<t<0$. This can give changes in the pole behavior or can lead to an effective discontinuity $\Delta T_{a}$.

For $k>1$, the dimensions of the pole terms change from $k-1$ to $k$ in the boundary region; this variation does not affect the fields of the reduced problem. For $k=1$, this rapid variation gives an effective discontinuity between the Nahm data on the two sides of the boundary in the large $D$ limit. The construction equation for this case has a solution localized in this small region that has the same effect as the jumping data in the reduced problem. For $k=0$, the rapid variation of $T_{a}^{L}$ cancels the effect of the original jumping data and makes the reduced Nahm data continuous across the boundary between the left and right intervals. For the $k<0$ case, removing a monopole on the left also causes changes on the right interval: The $|k|$-dimensional pole terms of the original $T_{a}^{R}$ are restricted to $0<t<\epsilon_{D}$ and go over to the $(|k|+1)$-dimensional pole terms of the reduced problem when $t>\epsilon_{D}$. As in the $k>1$ case, this small region has no effect on the fields.

In terms of the D-brane picture, removing massive fundamental monopoles corresponds to removing D-string segments. The transition between different types of boundary conditions can then be interpreted in terms of the interactions between the distant D-string segment and the rest of the system.

An interesting extension of this work would be to consider the case where some D3branes coincide with each other. In terms of the world-volume theory on the D3-branes, this corresponds to having a non-Abelian unbroken gauge symmetry. In these cases one finds solutions with clouds of non-Abelian fields surrounding one or more massive monopoles. These clouds can be interpreted as massless monopoles with non-Abelian magnetic charge [16, 17, 20,21,22, 23.

In terms of the Nahm data, these clouds have been found to arise in two different ways. In the first, the clouds originate from the pole behavior of the Nahm data. An example is the $([1], 2)$ solution 10 in $\mathrm{SU}(3) \rightarrow \mathrm{SU}(2) \times \mathrm{U}(1)$. The D-brane picture is sketched in Fig. 6, where $t_{\text {end }}=t_{1}=t_{2}$ is the position of the two coinciding D3-branes. The pole of the Nahm

\footnotetext{
${ }^{10}$ The square bracket denotes a massless monopole.
} 


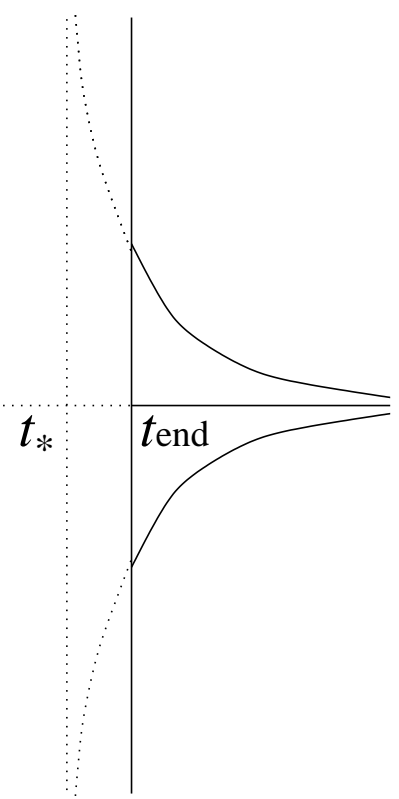

FIG. 6. The pole behavior for the non-Abelian cloud case. The dotted lines are extensions of the solid lines to the pole.

data is at $t_{*}<t_{\text {end }}$. Sending the massless monopole far away corresponds to bringing $t_{*}$ closer to $t_{\text {end }}$. The size of the cloud, which can be interpreted as the distance to the massless monopole, is approximately 23.

$$
a \approx \frac{1}{2 e\left(t_{\text {end }}-t_{*}\right)}
$$

We can understand this result heuristically by applying the large- $k$ D-brane analysis of Sec. VII to the present $k=2$ case. On the D3-branes, the non-Abelian Higgs fields of the known BPS solution 16,20 give $t-t_{\text {end }} \approx \frac{a}{2 e(r+a) r}$ for $a$ much greater than the massive monopole separation. For $a \gg r$ this gives

$$
r \approx \frac{1}{2 e\left(t-t_{\text {end }}\right)+1 / a}
$$

On the D-strings, since the pole position is at $t_{*}$, the D-string fluctuation scale

$$
R \approx \frac{1}{2 e} \frac{1}{\left(t-t_{\text {end }}\right)+\left(t_{\text {end }}-t_{*}\right)} .
$$

Setting $R=r$ then gives Eq. (8.1), up to a factor of $\mathcal{O}(1)$.

In the second case, the clouds arise from the jumping data. An example is the $(1,[1], 1)$ solution in $\mathrm{SU}(4) \rightarrow \mathrm{U}(1) \times \mathrm{SU}(2) \times \mathrm{U}(1)$. The relation between the cloud and the D-brane 
picture is less clear for this situation, as well as for the more complicated examples where both jumping data and pole behavior contribute to the clouds [23].

In this non-Abelian case the process of taking individual monopoles to spatial infinity is more complicated than when the symmetry is maximally broken. Because the long-range interactions between monopoles with non-Abelian charges are more complex than when only Abelian magnetic charges are present, it is not always possible to deform a solution into one that is essentially a superposition of widely separated component monopoles. A striking example of this is seen when one examines solutions with massive and massless component monopoles whose overall magnetic charge is purely Abelian. Previous studies 16, 17, 22, 23, of such "magnetically color-neutral" solutions have found that the massive monopoles must always be enclosed by one or more clouds. As a result, one cannot remove one of the massive monopoles to spatial infinity without simultaneously making one of the clouds infinite in size.

However, this is not necessarily the case for solutions that are not magnetically colorneutral. An example of this is provided by the solutions with charge ([1], $m$ ) for $\mathrm{SU}(3)$ broken to $\mathrm{SU}(2) \times \mathrm{U}(1)$. From the form of the Nahm construction for these solutions, it is easy to see that we can use the methods of this paper to remove $m-2$ of the $([0], 1)$ massive fundamental monopoles one by one, while still maintaining a finite size cloud. It is only when we are left with the color-neutral $([1], 2)$ solution that this procedure breaks down.

This process of removing massive monopoles can help us understand the parameters entering these solutions. Both the counting and the interpretation of these are more complicated than when all the monopoles are massive. With maximal symmetry breaking, the moduli space of $(1, m)$ solutions is $4(m+1)$-dimensional, with the parameters corresponding to three position and one $\mathrm{U}(1)$ collective coordinate for each of the massive monopoles.

With the non-maximal breaking, this counting still holds for the $m=2$ case, but the meaning of some of the parameters is changed. Eight are the usual position and U(1) parameters for the two massive monopoles, but three others correspond to global $\mathrm{SU}(2)$ rotations, while the last characterizes the size of the non-Abelian cloud. For $m \neq 2$, the dimensions of the spaces have been found to be 6 for $m=1$ and $4 m+6$ for $m>2$ [24]. (There is no solution for $m=0$.) By examination of the explicit $([1], 1)$ solutions [which are gauge equivalent to the $([0], 1)$ ones] one sees that four parameters are position and $\mathrm{U}(1)$ variables, while the remaining two dimensions are due to global $\mathrm{SU}(2)$ rotations that correspond to non-normalizable zero modes. To understand the parameters when $m>2$, we apply the methods of this paper to deform the generic solution into one containing $m-2$ massive monopoles that are well separated from each other and from a ([1],2) color-neutral configuration. It then seems clear that the parameters should be understood as $4(m-2)$ 
position and $\mathrm{U}(1)$ variables for the isolated massive monopoles, the twelve described above for the ([1],2) configuration, and two more corresponding to non-normalizable global SU(2) modes.

The generalization of this procedure to other charges and groups, as well as to solutions containing more than one cloud, remains a challenge for future work.

\section{ACKNOWLEDGMENTS}

This work was supported in part by the U.S. Department of Energy.

\section{APPENDIX A}

In this appendix we describe the details of the analysis of the Nahm data and solutions of the construction equation for the $k>1$ case described in Sec. IVA. We will study the case of charge $(m+k, m)$ with one $(1,0)$ monopole removed by a distance $D$, and compare it with the case of charge $(m+k-1, m)$. We will focus on the behavior in the neighborhood of the middle boundary at $t_{2}=0$, ignoring the boundary regions near $t_{1}$ and $t_{3}$.

We decompose the Nahm data $T_{a}^{L}$ and construction equation solution $v$ as

$$
T_{a}^{L}=\left(\begin{array}{ccc}
N_{a} & E_{a}^{\dagger} & F_{a}^{\dagger} \\
E_{a} & P_{a} & G_{a}^{\dagger} \\
F_{a} & G_{a} & b_{a}
\end{array}\right), \quad v=\left(\begin{array}{c}
u \\
w \\
z
\end{array}\right),
$$

where in each case the first entry is $m$-dimensional, the second is $(k-1)$-dimensional and the third is one-dimensional.

The Nahm equations separate into

$$
\begin{aligned}
& \frac{d N_{a}}{d t}=-i \epsilon_{a b c}\left(N_{b} N_{c}+E_{b}^{\dagger} E_{c}+F_{b}^{\dagger} F_{c}^{\dagger}\right), \\
& \frac{d E_{a}}{d t}=-i \epsilon_{a b c}\left(E_{b} N_{c}+P_{b} E_{c}+G_{b}^{\dagger} F_{c}\right), \\
& \frac{d F_{a}}{d t}=-i \epsilon_{a b c}\left(F_{b} N_{c}+G_{b} E_{c}+b_{b} F_{c}\right), \\
& \frac{d P_{a}}{d t}=-i \epsilon_{a b c}\left(E_{b} E_{c}^{\dagger}+P_{b} P_{c}+G_{b}^{\dagger} G_{c}\right), \\
& \frac{d G_{a}}{d t}=-i \epsilon_{a b c}\left(F_{b} E_{c}^{\dagger}+G_{b} P_{c}+b_{b} G_{c}\right), \\
& \frac{d b_{a}}{d t}=-i \epsilon_{a b c}\left(F_{b} F_{c}^{\dagger}+G_{b} G_{c}^{\dagger}\right) .
\end{aligned}
$$

From Eq. (2.5) we see that in the interval $(-1 / D, 0)$ the matrix 


$$
\left(\begin{array}{cc}
P_{a} & G_{a}^{\dagger} \\
G_{a} & b_{a}
\end{array}\right) \approx-\frac{J_{a}^{(k)}}{t},
$$

where the $J_{a}^{(k)}$ are a $k$-dimensional irreducible representation of $\mathrm{SU}(2)$. The behavior of the other elements of the $T_{a}^{L}$ in this region can also be seen from Eq. (2.5). For $t \leq-1 / l$, we have $b_{3}=D$ and the other elements $\mathcal{O}(l)$, just as in Sec. [II].

By a unitary transformation using

$$
U=\left(\begin{array}{ccc}
I_{m \times m}+\mathcal{O}\left(l^{2} / D^{2}\right) & \mathcal{O}\left(l^{2} / D^{2}\right) & -\mathcal{F}_{3}^{\dagger} / D \\
\mathcal{O}\left(l^{2} / D^{2}\right) & I_{(k-1) \times(k-1)}+\mathcal{O}\left(l^{2} / D^{2}\right) & -\mathcal{G}_{3}^{\dagger} / D \\
\mathcal{F}_{3} / D & \mathcal{G}_{3} / D & 1+\mathcal{O}\left(l^{2} / D^{2}\right)
\end{array}\right)
$$

we can subtract constants $\mathcal{F}_{3}$ and $\mathcal{G}_{3}$ from $F_{3}$ and $G_{3}$, respectively. We choose these constants so that $F_{3}$ and $G_{3}$ are exponentially small in the middle of the interval. Since, as we will see, $\mathcal{F}_{3}$ and $\mathcal{G}_{3}$ are both $\mathcal{O}(l)$, the effects of the transformation on the other elements of the $T_{a}^{L}$ are negligible.

Using arguments similar to those applied to the $A_{a}$ in Sec. III], we can show that for $t<-1 / D$ the first and second components of Eq. (A6) are dominated by the $b_{3} G_{c}$ term. This gives the $t$-dependence of the $G_{1,2}$ to be $\mathcal{O}(D) e^{-D|t|}$, where the coefficient must be $\mathcal{O}(D)$ so that it can match on to the pole at $t=-1 / D$. This, together with the third component of Eq. (A6]), gives $G_{3} \sim \mathcal{O}(l) e^{-D|t|}$. The first and second components of Eq. (A4) are dominated by the $G_{1,2} E_{c}$ or $b_{3} F_{c}$ terms. These give $F_{1,2} \sim \mathcal{O}(l) e^{-D|t|}$. The third component of Eq. (A4) then implies $F_{3} \sim \mathcal{O}(l) e^{-D|t|}$.

The orders of magnitude of $G_{1,2}$ justify our previous statement that $\mathcal{F}_{3}$ and $\mathcal{G}_{3}$ are at most $\mathcal{O}(l)$, because otherwise Eq. (A3) or Eq. (A7) would imply that $E_{1,2}$ or $b_{1,2}$ would be too big.

For $t<-t_{D}$, the $F_{a}$ and $G_{a}$ are exponentially small and can be ignored in Eqs. (A2), (A3), and (A5). These three are the Nahm equations for the $(m+k-1)$-monopole case. The poles of the matrices $P_{a}$ in $(-1 / D, 0)$ extend into this region and dominate the right-hand sides of Eqs. (A3) and (A5). This gives the $P_{a}$ poles whose residue is a $(k-1)$-dimensional irreducible $\mathrm{SU}(2)$ representation in $\left(-1 / l,-t_{D}\right)$. As in Eq. (2.5), we have $E_{a} \sim t^{(k-2) / 2}$. Finally Eq. (A7) can be used to refine the behavior of $b_{a}$ using the above information. We find that $b_{1,2}=\mathcal{O}(l)+\mathcal{O}(l) e^{-2 D|t|}$ and $b_{3}=D+\mathcal{O}(D) e^{-2 D|t|}$.

These results are summarized in Table A. 


\begin{tabular}{|c|c|c|c|c|c|}
\hline & $-1 / l$ & & $-t_{D}$ & $-1 / D$ & 0 \\
\hline$\overline{N_{a}}$ & $\mid \leftarrow$ & & $\mathcal{O}(l)$ & & $\rightarrow \mid$ \\
\hline$E_{a}$ & $|\leftarrow \mathcal{O}(l) \rightarrow| \leftarrow$ & $\mathcal{O}\left(t^{(k-2) / 2}\right)$ & $\rightarrow \mid \leftarrow$ transition & $\rightarrow \mid \leftarrow \mathcal{O}\left(t^{(k-1) / 2}\right)$ & $\rightarrow \mid$ \\
\hline$F_{a}$ & $\mid \leftarrow$ & $\mathcal{O}(l) e^{-D|t|}$ & $\rightarrow \mid \leftarrow$ transition & $\rightarrow \mid \leftarrow \mathcal{O}\left(t^{(k-1) / 2}\right)$ & $\rightarrow 1$ \\
\hline$P_{a}$ & $|\leftarrow \mathcal{O}(l) \rightarrow| \leftarrow$ & $-J_{a}^{(k-1)} / t$ & $\rightarrow \mid \leftarrow$ transition & $\rightarrow \mid \leftarrow \quad-J_{a}^{(k)} / t$ & $\rightarrow \mid$ \\
\hline$G_{1,2}$ & $\mid \leftarrow$ & $\mathcal{O}(D) e^{-D|t|}$ & $\rightarrow \mid \leftarrow$ transition & $-J_{a}^{(k)} / t$ & $\rightarrow \mid$ \\
\hline$G_{3}$ & $\mid \leftarrow$ & $\mathcal{O}(l) e^{-D|t|}$ & $\rightarrow \mid \leftarrow$ transition & $-J_{a}^{(k)} / t$ & $\rightarrow \mid$ \\
\hline$b_{1,2}$ & $\mid \leftarrow$ & $\mathcal{O}(l)+\mathcal{O}(l) e^{-2 D|t|}$ & $\rightarrow \mid \leftarrow$ transition & $-J_{a}^{(k)} / t$ & $\rightarrow \mid$ \\
\hline$b_{3}$ & $\mid \leftarrow$ & $D+\mathcal{O}(D) e^{-2 D|t|}$ & $\rightarrow \mid \leftarrow$ transition & $-J_{a}^{(k)} / t$ & $\rightarrow 1$ \\
\hline
\end{tabular}

Table A: The behavior of the elements in the Nahm matrices for the $k>1$ case. $J_{a}^{(k)}$ is a $k$-dimensional irreducible $\mathrm{SU}(2)$ representation. The value $-J_{a}^{(k)} / t$ in the last five entries indicates that the $P_{a}, G_{a}$ and $b_{a}$ together form a pole term $-J_{a}^{(k)} / t$.

From Eq. (A2) we see that the variation in the $N_{a}$ in the interval $\left(-t_{D}, 0\right)$ is negligible. Since the only boundary values of $N_{a}$ are required to connect to the next interval, this is what we need to show the equivalence of the Nahm data between the original and the reduced problem.

Next we turn to the construction equation. Using the notation of Eq. (A1), we have

$$
\begin{aligned}
& 0=-\frac{d}{d t} u+\left[\left(-N_{a}+r_{a}\right) \otimes \sigma_{a}\right] u-\left[E_{a}^{\dagger} \otimes \sigma_{a}\right] w-\left[F_{a}^{\dagger} \otimes \sigma_{a}\right] z, \\
& 0=-\frac{d}{d t} w-\left[E_{a} \otimes \sigma_{a}\right] u+\left[\left(-P_{a}+r_{a}\right) \otimes \sigma_{a}\right] w-\left[G_{a}^{\dagger} \otimes \sigma_{a}\right] z, \\
& 0=-\frac{d}{d t} z-\left[F_{a} \otimes \sigma_{a}\right] u-\left[G_{a} \otimes \sigma_{a}\right] w+\left[\left(-b_{a}+r_{a}\right) \otimes \sigma_{a}\right] z .
\end{aligned}
$$

As in Sec. [II], there are three types of solutions away from the boundaries. The second and third types of solutions can be ignored for the same reason as in that section. Here we study in more detail the first type of solution. In $(-1 / D, 0)$, these are normalizable with positive power dependence on $t$, as required in the $(m+k)$-monopole construction. Using this information and the order of magnitude of the $G_{a}$ from Table A, we see from the third term of Eq. (A12) that the $z$ component, which is exponentially small away from the boundary, can be $\mathcal{O}(w)$ in $\left(-t_{D}, 0\right)$. The orders of magnitude of the $P_{a}$ and the $G_{a}$ can also cause $\mathcal{O}(w)$ changes in the $w$ components in $\left(-t_{D}, 0\right)$. However, because of the smaller orders of magnitude of the $E_{a}$ and the $F_{a}$, the $u$ component of this type of solution is essentially unchanged in $\left(-t_{D}, 0\right)$. 
So, comparing the original and the reduced problem, we see that the $u$ component, including its boundary value, is unchanged in the $D \rightarrow \infty$ limit. The $w$ only differ by an amount of $\mathcal{O}(w)$ within $\left(-t_{D}, 0\right)$; this has no effect on the normalization condition, Eq. (2.3), and the fields, Eq. (2.4), in the limit. The boundary values for $w$ are always zero, as we can see from the its power dependence on $t$. The $z$ component is small except for being $\mathcal{O}(w)$ in a small region near the boundary, and can also be neglected in Eqs. (2.3) and (2.4). 


\section{REFERENCES}

[1] M. F. Atiyah, N. J. Hitchin, V. G. Drinfeld and Y. I. Manin, Phys. Lett. A 65, 185 (1978).

[2] W. Nahm, Phys. Lett. B 90, 413 (1980); W. Nahm, in Monopoles in Quantum Field Theory, edited by N. Craigie et al. (World Scientific, Singapore, 1982); W. Nahm, in Group Theoretical Methods in Physics, edited by G. Denardo et al. (Springer-Verlag, Berlin, 1984).

[3] E. B. Bogomol'nyi, Yad. Fiz. 24,861 (1976) [Sov. J. Nucl. Phy. 24, 449 (1976)];

M. K. Prasad and C. M. Sommerfield, Phys. Rev. Lett. 35, 760 (1975).

[4] E. J. Weinberg, Nucl. Phys. B 167, 500 (1980).

[5] E. Witten, J. Geom. Phys. 15, 215 (1995) arXiv:hep-th/9410052.

[6] M. R. Douglas, J. Geom. Phys. 28, 255 (1998) arXiv:hep-th/9604198.

[7] D. E. Diaconescu, Nucl. Phys. B 503, 220 (1997) arXiv:hep-th/9608163.

[8] A. Kapustin and S. Sethi, Adv. Theor. Math. Phys. 2, 571 (1998) arXiv:hep-th/9804027.

[9] D. Tsimpis, Phys. Lett. B 433, 287 (1998) arXiv:hep-th/9804081.

[10] C. G. Callan and J. M. Maldacena, Nucl. Phys. B 513, 198 (1998) arXiv:hep-th/9708147.

[11] G. W. Gibbons, Nucl. Phys. B 514, 603 (1998) arXiv:hep-th/9709027.

[12] J. Hurtubise and M. K. Murray, Commun. Math. Phys. 122, 35 (1989).

[13] N. J. Hitchin, Commun. Math. Phys. 89, 145 (1983).

[14] E. J. Weinberg, Phys. Rev. D 20, 936 (1979).

[15] C. Houghton, P. W. Irwin and A. J. Mountain, JHEP 9904, 029 (1999) arXiv:hepth/9902111.

[16] A. S. Dancer, Commun. Math. Phys. 158, 545 (1993). A. S. Dancer, Nonlinearity, 5 (1992).

[17] K. Lee and C. Lu, Phys. Rev. D 57, 5260 (1998) arXiv:hep-th/9709080.

[18] N. R. Constable, R. C. Myers and O. Tafjord, Phys. Rev. D 61, 106009 (2000) arXiv:hepth/9911136.

[19] D. Kabat and W. I. Taylor, Adv. Theor. Math. Phys. 2, 181 (1998) arXiv:hepth/9711078. S. J. Rey, arXiv:hep-th/9711081.

[20] E. J. Weinberg, Phys. Lett. B 119, 151 (1982).

[21] K. Lee, E. J. Weinberg and P. Yi, Phys. Rev. D 54, 6351 (1996) arXiv:hep-th/9605229.

[22] E. J. Weinberg and P. Yi, Phys. Rev. D 58, 046001 (1998) arXiv:hep-th/9803164.

[23] C. J. Houghton and E. J. Weinberg, Phys. Rev. D 66, 125002 (2002) arXiv:hepth/0207141.

[24] M. Murray, Commun. Math. Phys. 125, 661 (1989). 